\title{
PHOTOEVAPORATION OF CIRCUMSTELLAR DISKS DUE TO EXTERNAL FUV RADIATION IN STELLAR AGGREGATES
}

\author{
Fred C. Adams ${ }^{1,2}$, David Hollenbach ${ }^{3}$, Gregory Laughlin ${ }^{4}$, Uma Gorti ${ }^{3}$ \\ ${ }^{1}$ Michigan Center for Theoretical Physics, Physics Department \\ University of Michigan, Ann Arbor, MI 48109 \\ ${ }^{2}$ Astronomy Department, University of Michigan, Ann Arbor, MI 48109 \\ ${ }^{3}$ NASA Ames Research Center, Moffett Field, CA 94035 \\ ${ }^{4}$ Lick Observatory, University of California, Santa Cruz, CA 95064
}

\begin{abstract}
When stars form within small groups (with $N_{\star} \approx 100-500$ members), their circumstellar disks are exposed to relatively little EUV $(h \nu>13.6 \mathrm{eV})$ radiation but a great deal of FUV $(6 \mathrm{eV}<h \nu<13.6 \mathrm{eV})$ radiation $\left(\sim 10^{3}\right.$ times the local interstellar FUV field) from the most massive stars in the group. This paper calculates the mass loss rates and evaporation time scales for circumstellar disks exposed to external FUV radiation. Previous work treated large disks and/or intense radiation fields in which the disk radius $r_{d}$ exceeds the critical radius $r_{g}$ where the sound speed in the FUV heated surface layer exceeds the escape speed; it has often been assumed that photoevaporation occurs for $r_{d}>r_{g}$ and is negligible for $r_{d}<r_{g}$. Since $r_{g} \gtrsim 100$ AU for FUV heating, this would imply little mass loss from the planet-forming regions of a disk. In this paper, we focus on systems in which photoevaporation is suppressed because $r_{d}<r_{g}$ and show that significant mass loss still takes place as long as $r_{d} / r_{g} \gtrsim 0.1-0.2$. Some of the gas extends beyond the disk edge (or above the disk surface) to larger distances where the temperature is higher, the escape speed is lower, and an outflow develops. The resulting evaporation rate is a sensitive function of the central stellar mass and disk radius, which determine the escape speed, and the external FUV flux, which determines the temperature structure of the surfaces layers and outflowing gas. Disks around red dwarfs, low mass stars with $M_{*} \lesssim 0.5 M_{\odot}$, are evaporated and shrink to disk radii $r_{d} \lesssim 15 \mathrm{AU}$ on short time scales $t \lesssim 10 \mathrm{Myr}$ when exposed to moderate FUV fields with $G_{0}=3000$ (where $G_{0}=1.7$ for the local interstellar FUV field). The disks around solar type stars are more durable. For intense FUV radiation fields with $G_{0}=30,000$, however, even these disks shrink to $r_{d} \lesssim 15 \mathrm{AU}$ on time scales $t \sim 10 \mathrm{Myr}$. Such fields exist within about 0.7 pc of the center of a cluster with $N_{\star} \approx 4000$ stars. If our solar system formed in the presence of such strong FUV radiation fields, this mechanism could explain why Neptune and Uranus in our solar system are gas poor, whereas Jupiter and Saturn are
\end{abstract}


relatively gas rich. This mechanism for photoevaporation can also limit the production of Kuiper belt objects and can suppress giant planet formation in sufficiently large clusters, such as the Hyades, especially for disks associated with low mass stars.

\section{INTRODUCTION}

The collapse of molecular cloud cores leads to the formation of stars with orbiting accretion disks. The dust in these disks can settle, coagulate, and form solid objects ranging in sizes from pebbles to planetesimals to planets. However, a number of mechanisms act to disperse gas from these disks, either driving the gas back out into the interstellar medium, or spiraling it into the central star. During this dispersal, the gas can, in turn, drag small dust particles (with radii $b \lesssim 1$ $\mathrm{cm}$ ) along in the flow. Gas dispersal thus disrupts planet formation in at least two important ways: (i) If the gas is dispersed before the disk dust particles have coagulated to sizes sufficient to decouple from the gas flow $(b \gtrsim 1 \mathrm{~cm})$, then the formation of planetesimals, Kuiper Belt Objects, and rocky planets will be curtailed because all the orbiting solid material in the gas flow region is removed before it has a chance to grow. (ii) If the gas is dispersed before large $\left(m_{P} \gtrsim 5-15\right.$ earth masses) rocky planets are formed, and if giant planets form by the gravitational accretion of gas onto these large rocky cores (Bodenheimer \& Pollack 1986; Lissauer 1993), then the formation of gas giant planets like Jupiter and Saturn will be suppressed.

The dispersal of gas and small dust particles has other important effects on the formation of planetary systems. The presence of a moderately massive gas disk leads to planetary migration (Lin \& Papaloizou 1986, Ward 1997). The presence of even small amounts of gas at time scales $t \sim 10-100$ Myr after disk formation influences the dynamics and evolution of orbiting objects in solar systems. For example, such gas can affect the orbital eccentricities of both planets and any remaining planetesimals (e.g., Tanaka \& Ida 1997; Kominami \& Ida 2002; Chiang et al. 2002). Reducing the eccentricities can, in turn, alter the time required for the collisional agglomeration of large planets.

Observations of disk systems of various ages suggest that the small $(b \lesssim 1 \mathrm{~mm})$ dust particles disappear on time scales of roughly $3-10$ Myr (Haisch et al. 2001). Near infrared continuum observations probe dust orbiting in the central regions of disks, $r \lesssim 0.1 \mathrm{AU}$, whereas submillimeter and millimeter wavelength continuum observations probe dust in the outer disks, $r \gtrsim 30 \mathrm{AU}$. The small dust grains in both regions disappear on roughly the same time scale. Presumably, after the disappearance time scale, some of the small dust particles have coagulated to form pebbles and larger objects that are no longer detectable at IR or millimeter wavelengths. Several authors cite observational evidence for coagulated dust of size $b \sim 1 \mathrm{~cm}$ in young disks (d'Alessio et al. 1999, Throop et al. 2001). However, the present observations do not specify how much of the small dust has been dispersed, and how much has coagulated into larger objects and disappeared from view.

Observations of gas in disks indicate that gas can also be dispersed in a relatively short time 
of only $t \lesssim 10$ Myr (Zuckerman et al. 1995). Generally, the gas is traced by millimeter and submillimeter observations of the trace species ${ }^{12} \mathrm{CO}$ in the $\mathrm{J}=1-0,2-1$, or $3-2$ transitions, which are sensitive to low masses of gas as long as the gas disk is extended. Because these lines are optically thick, and beam dilution reduces the observed intensity for these small ( $\lesssim 1 ")$ disks, the current surveys are insensitive to gas of any mass at $r \lesssim 50$ AU for the nearby $(d \sim 100 \mathrm{pc}$ ) young star/disk systems. Nevertheless, significant gas masses have been detected via CO observations in disks as old as 10 Myr (cf. Carpenter 2002). In short, from an observational point of view, it appears that the bulk of the gas is dispersed from the outer disks in time scales $t \lesssim 10 \mathrm{Myr}$, but the evolution of the gas in the inner, planet-forming region of the disk is uncertain.

Hollenbach, Yorke, \& Johnstone (2000) reviewed theoretical models for dispersing the gas and small dust from disks. Observational evidence from our solar system and other planetary systems indicates that more gas and dust is accreted onto the central star and dispersed back into the ISM than forms planets or other solid orbiting objects. This dispersal is dominated by photoevaporation in the outer regions of disks and viscous evolution (accretion onto the star coupled with protostellar outflows) in the inner parts of disks. The boundary between these two regimes - viscous evolution and photoevaporation - remains uncertain. We need to develop a better understanding of viscous accretion and develop better photoevaporation models that accurately track mass loss at moderate radii from the central star. This paper addresses the latter problem for the case of external irradiation. Stellar winds may play a significant, but probably not dominant, role in dispersing gas at moderate radii near the boundary of the inner viscosity-dominated region and the outer photoevaporating region. Nearby stellar encounters, even for star/disks born in dense clusters like the Trapezium cluster, only affect the outermost regions $(r \gtrsim 100 \mathrm{AU})$ of the largest disks, and, even there, the photoevaporation of disks in these same clusters is likely to dominate the dispersal of the outer regions (e.g., Scally \& Clarke 2001, Clarke 2002, Adams \& Laughlin 2001).

Photoevaporation occurs when energetic photons heat the surface of the disk to elevated temperatures. The radiation of interest includes FUV photons in the energy range $6 \mathrm{eV}-13.6 \mathrm{eV}, \mathrm{EUV}$ photons in the energy range $13.6 \mathrm{eV}-100 \mathrm{eV}$, and $\mathrm{X}$-rays in the energy range $100 \mathrm{eV}-10 \mathrm{keV}$. If EUV photons can penetrate the outward flow and reach the disk surface, they will ionize and heat the surface to $T \approx 10^{4} \mathrm{~K}$, whereas the FUV and/or X-ray photons tend to heat the neutral gas to lower temperatures, typically in the range $100 \mathrm{~K}<T<3000 \mathrm{~K}$. The thermal pressures in these heated regions drive the gas outward and create a flow into the interstellar medium. An important critical radius $r_{g}$ can be defined - this fiducial length scale is the radius where the sound speed of the gas (hydrogen atoms) equals the escape speed from the gravitationally bound system, i.e.,

$$
r_{g}=\frac{G M_{*}\langle\mu\rangle}{k T} \approx 100 \mathrm{AU}\left(\frac{T}{1000 \mathrm{~K}}\right)^{-1}\left(\frac{M_{*}}{1 M_{\odot}}\right),
$$

where $M_{*}$ is the mass of the central star and $\langle\mu\rangle$ is the average mass of the gas particles. Most previous work on photoevaporation (Hollenbach et al. 1994, Johnstone et al. 1998, Störzer \& Hollenbach 1999) assumed that photoevaporation flow is only active for $r>r_{g}$, and that the disk is static (with a warm surface corona held in orbit by the stellar gravity) for $r<r_{g}$. In this paper, 
we generalize this picture to include a more proper treatment of the flow hydrodynamics and show that significant photoevaporation can take place for smaller radii, $r \gtrsim 0.2 r_{g}$ (see Figure 1 ). In any event, disk photoevaporation can be considered like a slow $\left(v \sim 1-5 \mathrm{~km} \mathrm{~s}^{-1}\right)$ thermal Parker wind originating from the outer portion of the disk $(r \sim 3-100 \mathrm{AU})$.

Other authors have discussed the possibility of significant flow from $r<r_{g}$ for disks surrounding compact objects (e.g., Begelman, McKee \& Shields 1983; Woods et al. 1996). This work showed that significant photoevaporation can take place outwards from $r \sim 0.2 r_{g}$ for the case of X-ray heated disks around black holes. Recently, Liffman (2003) presented an analytic argument for photoevaporative flow inside of $r_{g}$. Flow inside $r_{g}$ is important for protoplanetary disks. Often the heating raises the disk surfaces to $T \lesssim 1000 \mathrm{~K}$, so that $r_{g} \gtrsim 100$ AU. If photoevaporative flows are still significant at $r \sim 0.2 r_{g}$, then photoevaporation can effectively remove gas and dust from the region near $20 \mathrm{AU}$ and thereby affect the formation of Uranus, Neptune, and Kuiper Belt Objects in our solar system.

Photoevaporation can be initiated by the energetic photons from the central star or from a nearby, more massive, and luminous star in the stellar birth cluster. Hollenbach et al. (1994) originally modeled the evaporation caused by EUV photons from the central star. Johnstone, Hollenbach \& Bally (1998) and Störzer \& Hollenbach (1999) presented the first semi-analytic models of disks (with $r_{d}>r_{g}$ ) around low mass stars being photoevaporated by the FUV and EUV fluxes from a nearby OB star. These models were successfully applied to the PROtoPLanetarY DiskS, or "proplyds", observed in the cluster of low mass stars around the Trapezium in Orion (e.g., O'Dell 1998, Bally et al. 1998, Churchwell et al. 1987). A complementary set of models (Richling \& Yorke 1997, 1998, 2000; Yorke \& Richling 2002) studied the hydrodynamical flow for disks subjected to both radiation from their central stars and external radiation. This previous work produced two results of interest here: (i) In the case of external illumination, the FUV photons often initiate the mass loss and the incident EUV flux is absorbed at an ionization front in the neutral flow which is several disk radii away from the disk surface. (ii) The externally-illuminated disks evaporate from outside in, whereas the bulk of the mass loss for internally-EUV-illuminated disks occurs at $r \sim r_{g}$. In other words, in the former (external) case, a disk with outer radius $r_{d}$ shrinks from $r_{d}>r_{g}$ to $r_{d} \lesssim r_{g}$ as evaporation proceeds. In the latter case, in the absence of turbulent viscosity to drive radial flow and replenish material at $r_{g}$, the disk evaporates at $r_{g}$ until a gap is formed there, and then the photoevaporation proceeds from $r_{g}$ outward to $r_{d}$. These early models effectively assumed large disks with $r_{d}>r_{g}$. This paper presents a more in depth treatment for the case of small disks (with $r_{d}<r_{g}$ ) that are externally illuminated by FUV radiation. However, this work also has important implications for photoevaporation at $r<r_{g}$ for the internally-illuminated disks.

Several recent papers combine these early photoevaporation models with models of viscous accretion in attempts to model the time evolution of the dispersal of the entire protoplanetary disk. Clarke et al. (2001) treat the EUV photoevaporation by the central star coupled with viscous accretion and evolution to explain why disks are observed to rapidly disperse at the end of their lives on a time scale that is a small fraction of the disk lifetime. Matsuyama et al. (2003ab) model 
disks with both internal EUV and external FUV and EUV illumination and with viscous evolution. Such models will need modification in light of the results of this paper.

Stars often form in groups or clusters. If these stellar aggregates are large enough $\left(N_{\star} \gtrsim 200\right.$ stars), the system has a good chance of containing at least one O or early B star. In such systems, the low mass stars in the cluster are subject to significant photoevaporation by the FUV flux from other, larger stellar members. The ultimate goal for the work presented in this paper is to calculate the probability that a given low-mass star/disk system in the Galaxy, like the early solar nebula, experienced sufficient external illumination and consequent photoevaporation to affect the formation of gas-giant planets and/or the formation of planetesimals and planets in the Kuiper Belt region. Such a calculation would require the knowledge of the probability of being born in a cluster of given size $N_{\star}$ (e.g., see Lada \& Lada 2003, Porras et al. 2003, Adams \& Myers 2001, Carpenter 2000), the probability that one or several high mass stars are members of this cluster (given by the stellar initial mass function), the delay time between low mass star formation and high mass star formation, and the fraction of time that the low mass stars lie at a given distance from the unembedded OB star. Armitage (2000) presents a first attempt at such a model, which assumes that EUV-induced photoevaporation operates only for $r_{d}>r_{g}$. This paper presents the corresponding analysis for the case of FUV-induced photoevaporation that occurs for $r_{d}<r_{g}$; this latter process often dominates the mass loss for typical disks in typical star formation environments.

This paper is organized as follows. In $\S 2$, we discuss the physical mechanisms in photoevaporating disks, including heating processes, dust properties and attenuation, cooling mechanisms, thermal balance, and chemistry. We then summarize in more detail (in §3) the previous results for the photoevaporation of "large" (supercritical) disks with $r_{d}>r_{g}$, since these analytic results will be useful for generalization to the case of smaller disks. In $\S 4$, we calculate the photoevaporative mass loss rates and time scales for subcritical disks (with $r_{d}<r_{g}$ ) due to external FUV illumination. In general, photoevaporation takes place on both the disk surface, creating an initially vertical flow, and from the disk edge at $r_{d}$, creating a radial flow. Although the disk edge has less area, the radial flow tends to dominate the mass loss because the material here is bound more weakly. In a previous paper (Hollenbach \& Adams 2003), we presented the isothermal case, where we can obtain analytic approximations which provide physical insight; here we develop the more complicated (but more realistic) non-isothermal case where the temperature is determined from the heating and cooling of the gas in the flow. We determine how the mass loss rate depends on the incident FUV flux, the size $r_{d}$ of the disk, and the mass of the central star. We apply these results to the possible evaporation of the early solar nebula $(\S 5)$, the formation of Kuiper Belt objects and debris dust $(\S 6)$, the suppression of giant planet formation in large clusters like the Hyades $(\S 7)$, and the evaporation of disks around low mass stars $(\S 7)$. We conclude, in $\S 8$, with a summary and discussion of our results. 


\section{PHYSICAL MECHANISMS IN PHOTOEVAPORATING DISKS}

\subsection{Overview}

In an ideal case, one would solve the photoevaporation problem using a full three-dimensional treatment of the hydrodynamics, including time-dependent heating, cooling, and chemistry. Unfortunately, however, such a calculation is beyond the scope of this initial effort. Instead, we numerically solve the streamline equation for the flow hydrodynamics (in the spherical approximation) by utilizing temperatures derived from a state-of-the-art photodissociation region (PDR) code (Kaufman et al. 1999). This code self-consistently solves for the chemical abundances and gas temperature at any position (defined by the column density of hydrogen $N_{H}$ from that position to the FUV source) and for a given hydrogen gas density $n$. The PDR code assumes that thermal balance (heating and cooling rates are equal) and steady state chemical abundances have been achieved. We can check, post facto, that the flow time scales are long enough to justify these approximations. As we discuss below, when the flow approaches the sonic point, the ratio of the flow time to the heating time becomes smaller. We assume here that the gas temperature approaches a constant value near the sonic point and in the outer region of the flow.

In order to outline the physical mechanisms that operate during photoevaporation, we must define the basic flow quantities and their benchmark values. The outer radius of the disk $r_{d}$ marks the inner boundary of the flow (and the inner boundary of our calculations); we are primarily interested in disks of roughly solar system size, with $r_{d}=20-60 \mathrm{AU}$, and with moderate FUV heating so that $r_{d}<r_{g}$. The flow begins subsonically, with $v \ll a_{S}$, at the inner boundary $r_{d}$. As the gas flows outwards, the temperature and hence the sound speed increases. The flow speed increases more rapidly so that the the Mach number $\mathcal{M}=v / a_{S}$ increases and the flow reaches a sonic point. The radius $r_{s}$ of the sonic point is typically comparable to (but smaller than) the fiducial radius $r_{g}$ defined above (eq. [1]). For typical cases considered here (see $\S 4$ ), the sonic radius is a few times the outer disk edge and the critical radius is a few times the sonic radius. This paper thus works in the (previously unstudied) regime where $r_{d} / r_{g} \sim 0.2$ and finds that substantially mass loss can still take place for moderate values of the external FUV radiation field.

The results of this paper are primarily applicable to cases where a low mass star/disk system is formed within a small cluster or stellar group with $N_{\star}=100-500$ stars (see, e.g., Lada \& Lada 2003, Porras et al. 2003, Adams \& Myers 2001). In this setting, the disk will typically be illuminated by an $\mathrm{O}$ or $\mathrm{B}$ star which lies within the stellar birth aggregate, at a distance of 0.1 - 1 pc (e.g., Testi et al. 1997, 1998, 1999). For example, at a distance of 0.3 pc, a single main-sequence star of mass $M_{*}=8.7 M_{\odot}$ will produce an FUV radiation field with $G_{0} \approx 2000$ (Parravano, Hollenbach, \& McKee 2003). ${ }^{1}$ According to the standard initial mass function, a stellar

\footnotetext{
${ }^{1}$ Throughout this paper, we follow the standard convention of using the dimensionless parameter $G_{0}$ to measure the incident FUV flux. Specifically, $G_{0}=1$ corresponds to a radiative flux $1.6 \times 10^{-3} \mathrm{erg} \mathrm{cm}^{-2} \mathrm{~s}^{-1}$ in the $912 \AA$ to $2000 \AA$ band; this benchmark flux is typical of the local interstellar radiation field.
} 
aggregate with $N_{\star} \approx 200$ will have a 50-50 chance of producing a star this large (Adams \& Myers 2001). Notice that the total FUV radiation field produced by all the stars in the aggregate will be somewhat larger, so that $G_{0} \approx 3000$ is a reasonable benchmark value. We also stress that because stellar aggregates of this size will have relatively few massive stars, the radiation fields they produce will vary substantially from group to group due to incomplete sampling of the IMF.

These results also apply to low mass star/disk systems in large clusters, since even in the presence of EUV radiation, the FUV generally dominates the photoevaporation process until the disks shrink to sizes $r_{d} \lesssim 10-20$ AU (see Störzer \& Hollenbach 1999 and the discussion in §5). For a cluster containing $N_{\star} \approx 4000$ stars, similar to the Trapezium cluster, the OB stars will typically produce a field $G_{0} \approx 13,000 d_{\mathrm{pc}}^{-2}$, where $d_{\mathrm{pc}}$ is the distance in parsecs to the cluster center (Parravano 2003, private communication). Thus, for these large clusters, we take $G_{0} \approx 30,000$ as a benchmark value (applicable to stars in the cluster core with $d_{\mathrm{pc}}<1$ ).

Although this paper treats photoevaporation by calculating the depth dependent temperature of the gas, it is useful to keep in mind a simplified model of the photoevaporation mechanism: A surface layer of gas is heated to to a fixed temperature $T$ and a flow develops until the column density of the flow reaches a critical column density $N_{C}$. In this idealized model, the two flow parameters $\left(T, N_{C}\right)$, along with the stellar mass $M_{*}$ and disk radius $r_{d}$, completely specify the mass loss rate. As we discuss below, the column density is approximately that required to make the flow optically thick at FUV wavelengths so that $N_{C} \sim 10^{21} \mathrm{~cm}^{-2}$. The typical density at the base of the flow is about $n_{d} \sim 10^{7} \mathrm{~cm}^{-3}$, which is larger than, but comparable to the naive estimate $n \sim N_{C} / r_{d}$. The Appendix presents a modified version of the analytic treatment given in Hollenbach \& Adams (2003) for the photoevaporative mass loss rates from disks with $r_{d}<r_{g}$ (where the external heating is approximated by a semi-analytic model).

\subsection{Gas Heating Mechanisms}

Heating of the gas on the surface or edges of the disk ultimately causes the photoevaporative flow. The gas heating itself is driven by incident energetic photons. In this paper we focus on the mass loss driven by heating from FUV photons, although we note that EUV photons will also be present and can instigate additional mass loss (see the discussion in $§ 5$ ).

The FUV heating of the neutral gas at the disk surface and in the photoevaporative flow arises mainly from two mechanisms: grain photoelectric heating and the FUV pumping and subsequent collisional de-excitation of $\mathrm{H}_{2}$ molecules. In the former process, the FUV photons are absorbed by dust grains and a small fraction of these absorption events leads to the ejection of an energetic electron $(E \sim 1 \mathrm{eV})$ into the gas. As the electron collides with gas atoms and ions, it shares its kinetic energy as heat. In typical cases, about $1 \%$ of the absorbed FUV photon energy is delivered as gas heating through this mechanism. In the latter process, $\mathrm{H}_{2}$ molecules absorb FUV photons at particular electronic transition wavelengths in the range $912 \AA \leq \lambda \leq 1100 \AA$. These absorption 
events lead to electronic excitation of $\mathrm{H}_{2}$, followed by fluorescent decay to bound vibrational states of the ground electronic state ( $90 \%$ of the time). At the high densities at or near disk surfaces, this vibrational energy is converted to heat via collisional de-excitation by other hydrogen atoms or molecules (for further detail, see Hollenbach \& Tielens 1999).

\subsection{Dust Properties}

Dust plays several important roles in the photoevaporation process. The attenuation of the FUV photons by small $(b \sim 0.001-0.1 \mu \mathrm{m})$ dust particles carried along in the gas flow limits the depth of the FUV heating. Dust also provides heating processes which (in part) determine the gas temperature, either directly by grain photoelectric heating, or indirectly by providing the chemical catalyst that forms $\mathrm{H}_{2}$ (which affects both the heating and cooling rates).

Dust can be the agent that determines this critical column density $N_{C}$ (see $\S 2.1$ and Johnstone et al. 1998). In particular, dust properties determine $N_{C}$ for high ratios of the FUV flux to the gas density $G_{0} / n \gtrsim 10^{-2} \mathrm{~cm}^{3}$. Such high ratios ensure that $\mathrm{H}_{2}$ self-shielding is not very effective at the surface, so that the $\mathrm{H}_{2}$ abundance (and other molecular species that follow) increases only when the dust optical depth $\tau_{F U V}$ at FUV wavelengths becomes significant. In this case, $N_{C}=N_{F U V}$, the column density required for $\tau_{F U V}=1$, i.e., $N_{F U V} \sim 10^{21} \mathrm{~cm}^{-2}$. For larger optical depths, heating by FUV photons is less efficient and cooling rates via molecular species grow larger; as a result, the temperature drops precipitously.

The rough criterion that the dust optical depth of the flow is of order unity is easy to understand. If the column density $N_{H}$ from the base of the flow to the FUV source were small so that $\tau_{F U V} \ll 1$, then the FUV radiation would penetrate further and heat higher density gas deeper in the disk. This penetration would raise the column density $N_{H}$ and the corresponding optical depth $\tau_{F U V}$ of the flow. On the other hand, if $N_{H}$ were large so that $\tau_{F U V} \gg 1$, then FUV photons could not penetrate to the base of the flow, the gas would not be heated there, and the solution would be inconsistent because no flow could originate from such cold gas deep inside the disk.

In this work, we consider systems with lower FUV fluxes and higher gas densities than for cases considered previously (like the proplyds modeled in Johnstone et al. 1998), so that $G_{0} / n$ can be less than $10^{-2} \mathrm{~cm}^{3}$. In this regime, the $\mathrm{H}_{2}$ self shields and the gas becomes predominantly $\mathrm{H}_{2}$ at an optical depth less than $\tau_{F U V}=1$, or a column less than the benchmark value $N_{F U V} \sim 10^{21} \mathrm{~cm}^{-2}$. The gas then cools before the FUV is attenuated by dust; even though the heating rate remains high, the cooling rate is enhanced by the presence of molecules. As a result, the temperature in the atomic heated surface layer drops to more moderate values at a column density $N_{H}<N_{F U V}$, and then at $N_{H}=N_{F U V}$ the temperature drops further because of the loss of heating.

The dust abundances, and hence the dust opacities, affect all of the above considerations. Störzer \& Hollenbach (1999) modeled 10 proplyds in Orion and found that the best fit FUV dust cross section per $\mathrm{H}$ nucleus in these photoevaporative flows was approximately $\sigma_{F U V}=8 \times 10^{-22}$ 
$\mathrm{cm}^{2}$ per $\mathrm{H}$ nucleus, which is about 0.3 times the value for standard interstellar dust. This finding provides strong evidence for moderate coagulation and settling of the dust in the surfaces of these disks at distances $r \sim 30-100$ AU from the central star (for disk ages $\sim 1 \mathrm{Myr}$ ). We use this value of the FUV dust opacity for the models presented in this paper. We further discuss the coagulation of the dust in disks later in $\S 5$, where we apply our models to the formation of Kuiper Belt Objects and the possible presence of a sharp cutoff in Kuiper Belt Objects beyond 50 AU in the solar system.

\subsection{Gas Cooling Mechanisms}

The PDR code includes a large number of cooling mechanisms (see Tielens \& Hollenbach 1985, Kaufman et al. 1999). For most of the cases considered in this paper, where gas densities $n \approx 10^{4}-10^{8} \mathrm{~cm}^{-3}$ and $T \approx 100-3000 \mathrm{~K}$, the most important gas coolants include gas-grain collisions (the grains are typically much cooler than the gas, of order $T=10-50 \mathrm{~K}$ ), and collisional excitation followed by radiative decay of [CII] $158 \mu \mathrm{m}$, [OI] $63 \mu \mathrm{m}$, and the rotational and vibrational transitions of $\mathrm{H}_{2}, \mathrm{CO}$, and $\mathrm{OH}$. In rough terms, cooling by adiabatic expansion is only important when the flow time scale is relatively short compared to the heating time. In this setting, the flow is slow (subsonic) near the disk and supersonic in the outer region. We treat this outer regime by assuming that the flow has a constant temperature and constant flow speed for $r>r_{s}$.

\subsection{Thermal Balance}

The PDR code assumes thermal balance, i.e., that the sum of the heating rates is equal to the sum of the cooling rates. Given enough time, gas will reach this state of thermal balance. In this context, however, the gas is flowing outward and has a limited time to reach its preferred thermal state. To justify the assumption of thermal balance, we must compare the flow time with the heating time. The time scale $t_{f}$ for the flow to cross a radial scale $r$ is given by

$$
t_{f} \equiv r / v_{f} \simeq 95\left(\frac{r}{20 \mathrm{AU}}\right)\left(\frac{v_{f}}{1 \mathrm{~km} / \mathrm{s}}\right)^{-1} \text { years }
$$

where $v_{f}$ is the flow speed. The heating timescale $t_{h}$ is given by

$$
t_{h} \equiv n k T / \mathcal{H} \simeq 4.4\left(\frac{n}{10^{6} \mathrm{~cm}^{-3}}\right)\left(\frac{T}{10^{3} \mathrm{~K}}\right)\left(\frac{\mathcal{H}}{10^{-15} \mathrm{erg} \mathrm{cm}^{-3} \mathrm{~s}^{-1}}\right)^{-1} \text { years }
$$

where $\mathcal{H}$ is the heating rate per unit volume. To obtain the benchmark value of the heating rate, we have used a number density $n=10^{6} \mathrm{~cm}^{-3}$ and a radiation field with $G_{0}=3000$; keep in mind that $\mathcal{H}$ depends on both $n$ and $G_{0}$. For this case, the heating rate $\mathcal{H} \simeq 10^{-15} \mathrm{erg} \mathrm{cm}^{-2} \mathrm{~s}^{-1}$ and for hot $(T \simeq 1000 \mathrm{~K})$ surface gas, and we find that $t_{h}<t_{f}$. As a result, thermal balance is justified in this

fiducial case. We expect that the gas will be close to thermal balance in the inner regions where 
the flow is subsonic. In the outer region, however, the flow becomes supersonic and the heating time can exceed the dynamical flow time scale. In this paper, we take into account this effect by assuming that the gas temperature becomes isothermal in the outer region $r \geq r_{s}$. Specifically, we allow the temperature to increase, according to the heating/cooling treatment described here, from the inner boundary $r_{d}$ out to the sonic point $r_{s}$, and then assume that the temperature remains constant for $r>r_{s}$.

\subsection{Chemistry}

The PDR code includes 46 chemical species and 222 chemical reactions (Tielens \& Hollenbach 1985, Kaufman et al. 1999). The code is designed to follow the dominant coolants and includes substantial hydrogen, oxygen, and carbon chemistry, but only limited sulfur chemistry and essentially no nitrogen chemistry. The code calculates the steady state molecular abundances as a function of column density $N_{H}$ (or, equivalently, the visual extinction $A_{V}$ ). The molecular chemistry is quite complicated, but has been elucidated in previous work (again, see Kaufman et al. 1999). In the present context, the most important chemical activity is that the $\mathrm{H}_{2}$ abundance rises substantially once the column density from the disk surface exceeds $N_{H} \simeq 10^{20} \mathrm{~cm}^{-2}$. This formation of molecular hydrogen is the precursor to the formation of other molecules and it thus marks the onset of molecular cooling, which in turn leads to a drop in the gas temperature $T$ at large column density.

In the outflowing gas, the predominant chemistry involves the formation of molecules and their destruction by photodissociation. The chemical time scales for most molecules is comparable to the photodissociation time

$$
t_{\mathrm{chm}} \approx 0.1\left(\frac{G_{0}}{3000}\right)^{-1} \text { year }
$$

which is short compared to the flow time scale, thereby justifying the assumption of steady state. Although this time scale applies to most molecules, $\mathrm{H}_{2}$ is an important exception. Because $\mathrm{H}_{2}$ self shields, its photodissociation time scale is much longer. As a result, $\mathrm{H}_{2}$ can advect closer to the surface than the steady state model predicts (e.g., Bertoldi \& Draine 1996, Störzer \& Hollenbach 1998). The extra $\mathrm{H}_{2}$ increases the heating rate as well as the cooling rate. Although this effect could be significant, a detailed treatment is beyond the scope of this paper because it requires a timedependent PDR code coupled with a hydrodynamical calculation. The most important ramification of this effect is its signature in the temperature structure of the flow ( $T$ as a function of column density $N_{H}$ ). However, the results of Störzer \& Hollenbach (1998) indicate that the effects are relatively modest, so this paper still provides a good first approximation to the photoevaporation problem for $r_{d}<r_{g}$. 


\subsection{Temperature Profiles}

The net result of the PDR code calculations is a determination of the gas temperature as a function of both number density $n$ and visual extinction $A_{V}$ (or column density $N_{H}=\delta_{U V} A_{V} / \sigma_{F U V}$, where $\delta_{U V}=1.8$ is the conversion factor between visual extinction and FUV optical depth). The resulting temperature profiles are shown in Figure 2. Each panel corresponds to a given external radiation field. The temperature profiles, plotted as a function of $A_{V}$, are shown for each number density. Recall that the PDR code itself uses a constant density, plane parallel configuration and calculates the temperature by using a detailed treatment of heating, cooling, and chemistry. The key assumption in this work is that the resulting temperature dependences $T\left(n, A_{V}\right)$ provide a good working approximation for other geometrical configurations, in particular the radial flow fields that arise during the photoevaporation process (see $\S 3$ and 4 ).

Examination of the temperature profiles in Figure 2 shows a number of significant trends. For a given radiation field and a given density, the temperature approaches a nearly constant value at low visual extinction $A_{V}$. The temperature decreases slowly with increasing $A_{V}$ until the optical thickness of the dust becomes significant at $A_{V} \sim 1$. The temperature then decreases sharply as $A_{V}$ increases further. The outer temperature (at low $A_{V}$ ) generally increases with the intensity $G_{0}$ of the radiation field (as expected), although this outer temperature also varies significantly with the density. Finally, the temperature is not a smooth monotonic function of visual extinction $A_{V}$

or number density $n$. The temperature profiles exhibit a great deal of structure, mostly due to the heating and cooling effects of various molecules (and atoms) that come in and out of existence with varying $n$ and $A_{V}$.

\section{PHOTOEVAPORATION OF SUPERCRITICAL DISKS}

In this section, we review the simple models for photoevaporation that have been developed previously for the supercritical regime where $r_{d}>r_{g}$ (e.g., Johnstone et al. 1998). In this regime, the disk gas that resides at radii from $r_{d}$ to $r_{g}$ has little gravitational binding energy and readily achieves supersonic flow speeds near $r \sim r_{d}$. Because the disk edges have less surface area than the faces, most of the mass loss is driven off of the top and bottom disk surfaces (in contrast to the case of subcritical disks - see $\S 4$ ). These disks generally have surface density profiles that decrease with radius, and the disks shrink from the outside inwards as they evaporate in external FUV fields (this is also the case for subcritical disks).

The vertical flow off the disk follows pressure gradients that rapidly turn the flow into the radial direction by the time the flow reaches $r \gtrsim r_{d}$. Previous studies of this supercritical flow have generally worked in the limit $G_{0} / n \geq 10^{-2} \mathrm{~cm}^{3}$ where the flow column density $N_{H} \approx N_{F U V}=N_{C}$ (again, see Johnstone et al. 1998). In this regime, the condition $\tau_{F U V} \sim 1$ defines the total column density so that the outgoing flow itself is the limiting factor: As the mass outflow rate $\dot{M}$ increases, the optical depth of the flow increases and the $\tau_{F U V}=1$ limit is reached. If the flow were to 
increase beyond this level, the flow would become so optically thick that the driving FUV photons could no longer penetrate down to the disk.

These models also assume that the flow speed approaches a constant value in the region where most of the column density resides. With this assumption, in conjunction with radial symmetry, the continuity equation implies that the density field of the flow takes the form

$$
n(r)=n_{b}\left(r_{d} / r\right)^{2}
$$

where $n_{b}$ is the number density at the base of the flow. The total column density $N_{H}$ is given by the integral

$$
N_{H}=\int_{r_{d}}^{\infty} n(r) d r=n_{b} r_{d}
$$

The dust optical depth is given by $\tau_{F U V}=\sigma_{F U V} N_{H}$, where $\sigma_{F U V} \approx 8 \times 10^{-22} \mathrm{~cm}^{2}$ is the appropriate cross section for dust grains interacting with FUV radiation (e.g., see SH99). The optical depth unity surface thus defines a constraint on the base density $n_{b}$, i.e.,

$$
n_{b} r_{d}=\sigma_{F U V}{ }^{-1} \approx 10^{21} \mathrm{~cm}^{-2} .
$$

For the case of a large disk with $r_{d}>r_{g}$, the mass outflow rate is given by

$$
\dot{M}=\mathcal{F} 4 \pi r_{d}^{2} n_{b} a_{S}\langle\mu\rangle,
$$

where $\mathcal{F}$ is the fraction of the solid angle subtended by the outflow and $\langle\mu\rangle$ is the mass of the gas molecules (the conversion factor between number density and mass density). For $r_{d}>r_{g}$, the flow from the disk surface and the disk edge merge at roughly $r_{d}$ to $2 r_{d}$, creating a nearly spherically symmetric flow so that $\mathcal{F} \sim 1$. Using the $\tau_{F U V}=1$ constraint to define the value of the base density $n_{b}$, we obtain an estimate of the mass loss rate,

$$
\dot{M}=4 \pi \mathcal{F}\langle\mu\rangle \sigma_{F U V}{ }^{-1} a_{S} r_{d} \approx 1.2 \times 10^{-7} M_{\odot \mathrm{yr}^{-1}} \mathcal{F}\left(\frac{a_{S}}{2 \mathrm{~km} / \mathrm{s}}\right)\left(\frac{r_{d}}{100 \mathrm{AU}}\right),
$$

where everything is specified except for the disk radius $r_{d}$ and the sound speed $a_{S}$ of the flow $\left(a_{S}\right.$ is set by the temperature, which is set by the external radiation flux). In the second approximate equality, we have defined a benchmark evaporation rate for the supercritical regime using $r_{d}=100$ $\mathrm{AU}$ and $a_{S}=2 \mathrm{~km} / \mathrm{s}$ (for $T \approx 600 \mathrm{~K}$ ). When a typical solar nebula (with disk mass $M_{d}=0.03$ $M_{\odot}$ ) experiences mass loss in this supercritical regime, the evaporation time scale is only about 0.25 Myr, much less than the expected time scale for giant planet formation ( $t \sim 10$ Myr; e.g., Lissauer 1993). Thus, supercritical evaporation can readily evaporate nebular disks and compromise the planet formation process in the outer regions. However, for $M_{*}=1.0 M_{\odot}$ and $T=600 \mathrm{~K}$, the critical radius $r_{g}=160 \mathrm{AU}$ so that only the largest disks can experience supercritical mass loss. Many disks will live in the subcritical regime, and we must generalize this treatment, as outlined in the following section. 


\section{PHOTOEVAPORATION OF SUBCRITICAL DISKS}

In this section, we generalize the photoevaporation model to include cases where the disk radius is smaller than the critical radius, i.e., $r_{d}<r_{g}$. In this regime, the disk material is not immediately free to escape because the sound speed in the outer layer (that heated by FUV radiation) is still less than the escape speed. However, the disk has an atmosphere that extends beyond the nominal radius $r_{d}$ and some portion of that atmosphere will extend above the $r=r_{g}$ surface and can be susceptible to evaporation. As material leaves the system, an outward flow develops. The result is much like a Parker Wind solution: The flow starts subsonically at $r \ll r_{g}$, accelerates up to a sonic point at $r_{s} \lesssim r_{g}$, and then expands supersonically outwards. We thus need to make a simple model of the disk atmosphere and the accompanying flow.

\subsection{Basic Flow Geometry}

This problem contains four important length scales. In the supercritical regime considered previously (§3), the escape radius $r_{g}$ (eq. [1]) marks the inner boundary of the flow. In the subcritical regime considered here, the inner boundary of the flow is the disk radius $r_{d}$ where the flow speed is subsonic $\left(v \ll a_{d}\right)$. As the material flows outwards, the Mach number increases and the flow eventually exceeds the local sound speed at a sonic point $r_{s}$ (which marks the outer boundary of our numerical calculations). The sonic point $r_{s}$ is smaller than (but roughly comparable to) the escape radius $r_{g}$. The final length scale of interest is the disk scale height $H_{d}$ at $r_{d}$. If the disk extended out to the escape radius, then $H_{d} \sim r_{d} \sim r_{g}$; in the subcritical regime, however, the disk is relatively "thin" so that $H_{d}<r_{d}$. As a result, as shown in Figure 1, the basic length scales for these evaporating disks obey the ordering

$$
H_{d}<r_{d}<r_{s}<r_{g}
$$

The disk atmosphere behaves differently in the radial $(\hat{r})$ and vertical $(\hat{z})$ directions. As shown below and in the Appendix, the outflow from the disk edges (the radial flow) dominates the outflow from the disk faces (the vertical flow). As a result, we can assume that the essential part of the outflow takes place radially outwards from the disk edges. With this simplification, we construct a quasi-spherical disk model and take into account the fraction $\mathcal{F}$ of the solid angle that is subtended by the outflow from the disk edge (for a given scale height $H_{d}$ ). We also assume that radiation can hit the system at any angle, so that the disk receives its full quota of FUV radiation. A schematic diagram of this system is shown in Figure 1. Although the vertical flow is secondary in importance (for determining the outflow rate), the polar regions are not evacuated; these cavities will be filled by (more slowly moving) material that will attenuate the incoming FUV radiation.

To show that the radial flow tends to dominate the vertical flow, we consider the (one dimensional) profiles of density in the two directions in the hydrostatic limit. The density profile in the 
vertical direction can be written in the form

$$
\log n / n_{d}=-\frac{G M_{*}}{r_{d} a_{d}^{2}}\left[1-\frac{1}{1+z^{2} / r_{d}^{2}}\right]
$$

where we use an isothermal approximation so that $a_{d}$ is the isothermal sound speed at the disk surface. Note that this form does not assume small $z \ll r$. However, this equation is strictly valid for only the outermost annulus of the disk (at smaller disk radii, the gas is deeper in the potential well and contributes little to the mass outflow).

Similarly, we can integrate the hydrostatic force equation in the radial direction to obtain

$$
\log n / n_{d}=-\frac{G M_{*}}{2 r_{d} a_{d}^{2}}\left(1-\frac{r_{d}}{r}\right)^{2} .
$$

Since the vertical coordinate $z$ starts at $z=0$, and the radial coordinate starts at $r=r_{d}$, we define $x=r-r_{d}$ and rewrite the radial profile in the form

$$
\log n / n_{d}=-\frac{G M_{*}}{2 r_{d} a_{d}^{2}}\left(\frac{x}{r_{d}}\right)^{2}\left(\frac{1}{1+x / r_{d}}\right)^{2}
$$

A straightforward comparison shows that the right hand side of equation (11) is always greater than [or equal to - but only at the disk surface $(z=0)$ at $r_{d}$ (where $x=0$ )] the right hand side of equation (13). This result implies that the effective scale height of the density profile is always larger in the radial direction than in the vertical direction (so that the density falls off more slowly in $r$ ). Because the density tends to decrease more quickly in the vertical direction than in the radial direction, the density remaining at the sonic point will be greater for radial flow, and the mass loss rate will be larger for radial flow. In this paper, we thus assume that the radial portion of the flow dominates, and model the system using a quasi-spherical calculation. We also note that equation (13) for the radial density profile holds over the entire area of the disk edge, whereas equation (11) for vertical flow is only valid only for the outermost annulus of the disk (at $\left.r_{d}\right)$. At smaller radii, the gas is deeper in the potential and less likely to get out. As a result, the area of the disk edge has a greater working surface area than the disk face. This area argument thus argues that the radial flow from the disk edge is most important (see the Appendix for a more quantitative argument showing that radial flow dominates the vertical flow for $r_{d} \ll r_{g}$ ).

For these systems where the radial flow from the disk edge dominates the vertical flow from disk faces, we calculate the mass outflow rates by constructing a radial wind solution, but assume that only a fraction $\mathcal{F}$ of the solid angle is filled by the flow. The disk edges are essentially a cylinder of radius $r_{d}$ and height $2 H_{d}$, and thus subtend a given fraction of the $4 \pi$ steradians of solid angle centered on the star. Since the outflow is nearly radial, the solid angle subtended by the flow remains constant with radius and is given by

$$
\mathcal{F}=\frac{H_{d}}{\left(H_{d}^{2}+r_{d}^{2}\right)^{1 / 2}},
$$

where the disk scale height $H_{d} \approx r_{d} a_{d}\left(G M_{*} / r_{d}\right)^{-1 / 2}$. We note that the remaining solid angle is not evacuated. These regions contain slower moving material that will contribute to the attenuation of incoming FUV radiation, but will contribute relatively little to the total mass loss rate. 


\subsection{The Outer Region}

The flow in the outer region, beyond the sonic point, provides an outer boundary condition for the flow in the region of interest $\left(r_{d} \leq r \leq r_{s}\right)$. In the outer region where $r>r_{s}$, we assume the the flow is radial and has constant flow velocity. In other words, beyond the sonic point we assume that the flow has the same properties as found for the supercritical regime ( $\S 3$ ), albeit with lower mass loss rates $\dot{M}$. As a result, the density takes the form

$$
n_{\text {out }}=n_{s}\left(r_{s} / r\right)^{2}
$$

where $n_{s}$ is the number density at $r_{s}$. The column density $N_{s \infty}$ of the outer region is given by the integral

$$
N_{s \infty}=\int_{r_{s}}^{\infty} n_{\text {out }}(r) d r=n_{s} r_{s}
$$

and the corresponding dust optical depth of this region is given by

$$
\tau_{s \infty}=\sigma_{F U V} n_{s} r_{s}
$$

\subsection{Basic Equations of Motion}

If we include rotation for the force balance in the circumstellar disk, the radial force equation takes the form

$$
v \frac{d v}{d r}+\frac{1}{\rho} \frac{d P}{d r}+\frac{G M_{*}}{r^{2}}-\frac{j^{2}}{r^{3}}=0,
$$

where $j$ is the specific angular momentum. We can specify the angular momentum by requiring it to be the Keplerian value at the outer disk edge so that $j^{2}=G M_{*} r_{d}$. Now we assume an ideal gas law for the pressure, i.e., $P=n k T$. To simplify the equations, let $\xi=r / r_{d}, f=T / T_{d}, g=n / n_{d}$, and $u=v / a_{d}$ (where $a_{d}$ is the sound speed at the disk edge). The force equation becomes

$$
u \frac{d u}{d \xi}+\frac{1}{g} \frac{d}{d \xi}(g f)+\beta \frac{\xi-1}{\xi^{3}}=0
$$

where we have defined

$$
\beta \equiv \frac{G M_{*}\langle\mu\rangle}{k T_{d} r_{d}}=\frac{G M_{*}}{r_{d} a_{d}^{2}}
$$

The parameter $\beta$ can also be written in the form $\beta=r_{g} T_{g} / r_{d} T_{d}$ and thus provides a measure of how subcritical the disk edge is.

Now we introduce the continuity equation, which takes the form

$$
\dot{M}=4 \pi r^{2} \mathcal{F}\langle\mu\rangle n v=\text { constant },
$$

where we have included the filling factor $\mathcal{F}$. If we define a constant $C$ according to

$$
C \equiv\left(\frac{\dot{M}}{4 \pi r_{d}^{2} \mathcal{F}\langle\mu\rangle n_{d} a_{d}}\right)^{2}=\frac{v_{d}^{2}}{a_{d}^{2}},
$$


the dimensionless form of the continuity equation becomes

$$
\xi^{2} g u=\sqrt{C} .
$$

We can use the continuity equation to eliminate the flow speed $u$ from the differential equation (19), and thereby obtain a single differential equation (in density $n$ or $g=n / n_{d}$ ) to describe the flow. Alternately, we can use the continuity equation to eliminate the density from the force equation and obtain a differential equation for the flow speed. The former approach allows us to solve for the density structure of the flow. The latter approach defines the sonic point, which is necessary to define boundary conditions. So we follow both approaches.

\subsection{The Resulting Flow Equations}

By eliminating the flow speed $v$ through the continuity equation, the force equation becomes

$$
\frac{d}{d \xi}(g f)+\beta g \frac{\xi-1}{\xi^{3}}+\frac{C}{\xi^{2}} \frac{d}{d \xi}\left(\frac{1}{g \xi^{2}}\right)=0,
$$

where the constant $C$ is defined above. It is useful to expand this equation to obtain the form

$$
\frac{d g}{d \xi}\left(f-\frac{C}{\xi^{4} g^{2}}\right)=\frac{2 C}{\xi^{5} g}-\beta g \frac{\xi-1}{\xi^{3}}-g \frac{d f}{d \xi} .
$$

The PDR models specify the temperature as a function of column density $N_{H}$ or, equivalently, the visual extinction $A_{V}$. Here we can work in terms of the variable $\tau_{F U V} \equiv N_{H} \sigma_{F U V}$, which is a dust optical depth. To complete the specification of the problem (essentially, in order to determine the temperature), we need to include the differential equation that determines the optical depth $\tau_{F U V}$ as a function of $\xi$. In dimensionless form, $\tau_{F U V}$ is determined by the equation

$$
\frac{d \tau_{F U V}}{d \xi}=-\sigma_{F U V} r_{d} n_{d} g=-\tau_{d} g
$$

where the second equality defines $\tau_{d}=\sigma_{F U V} r_{d} n_{d}$.

Notice that the evaluation of $d f / d \xi$ is a bit subtle, since $f$ (the dimensionless temperature) is a function of both the density $(g)$ and the column density (or $\tau_{F U V}$ ). Thus, we can write

$$
\frac{d f}{d \xi}=\frac{1}{T_{d}} \frac{\partial T}{\partial n} \frac{d n}{d \xi}+\frac{1}{T_{d}} \frac{\partial T}{\partial \tau_{F U V}} \frac{d \tau_{F U V}}{d \xi} .
$$

Notice, however, that the definition of $d f / d \xi$ contains the derivative of the density $(d n / d \xi$ or $d g / d \xi)$ so that equation (25) remains in implicit form.

In this approach, the stellar mass $M_{*}$, radius $r_{d}$, and outer disk temperature $T_{d}$ are given system parameters, so the constant $\beta$ is specified. However, the density at the disk edge $n_{d}$ and the constant $C$ which determines the flow velocity are not determined in advance. The density $n_{d}$ 
enters into the problem by pinning down the scale for the column density. The constant $\sqrt{C}$ is the dimensionless mass outflow rate (i.e., $\dot{M}$ ), the quantity that we want to calculate in the end. For given estimates of $n_{d}$ and $C$, the differential equations (25) and (26), along with the definition (27), can be integrated outwards to the sonic point.

\subsection{Specification of the Sonic Point}

To determine the location of the sonic point, we need to eliminate the density from the force equation (instead of the velocity) by using the continuity equation. Using the same dimensionless formulation as before, we obtain

$$
\frac{1}{u} \frac{d u}{d \xi}\left(u^{2}-f\right)=\frac{2 f}{\xi}-\frac{d f}{d \xi}-\beta \frac{\xi-1}{\xi^{3}} .
$$

At the sonic point, $u^{2}=f$, the left hand side of the equation vanishes, and so the right hand side of the equation must vanish also. This constraint implies the relation

$$
2 f \xi^{2}-\beta(\xi-1)-\xi^{3} \frac{d f}{d \xi}=0
$$

which thereby defines the sonic point. The full definition of the sonic point thus involves a cubic equation in $\xi$. In practice, however, the final term is relatively small. Furthermore, because the flow time must be longer than the heating time in order for the outflowing gas to change its temperature,

the gas tends to become isothermal near the sonic point so that $f \rightarrow$ constant. As outlined above (see $\S 2$ ), we assume that the flow reaches both a constant temperature and a constant flow speed in the outer region. With this specification of our outer boundary condition, the sonic condition is the solution to equation (29), which becomes quadratic in the limit $d f / d \xi=0$, i.e.,

$$
\xi_{s}=\frac{\beta}{4 f}\left[1+(1-8 f / \beta)^{1 / 2}\right] .
$$

We have chosen here the physically realistic root of the quadratic equation, i.e., the root that has the form $\xi_{s} \rightarrow \beta / 2 f$ in the limit of large $\beta$. The other root approaches unity in this limit and is unphysical.

\subsection{Iteration Procedure}

If we specify the radiation field $G_{0}$, the disk size $r_{d}$, the outer disk temperature $T_{d}$, and the stellar mass $M_{*}$, then we need to solve self-consistently for the density $n_{d}$ at the base of the flow (our inner boundary) and the constant $C$ that sets the flow speed at the inner boundary or, equivalently, the dimensionless mass loss rate. In the absence of external radiation, the disk would have a density set by its temperature and surface density; the quantity $n_{d}$ is the density at the base of the outflow, 
which does occur at $r \sim r_{d}$, but may be a scale height or so above the original (not externally heated) disk itself. For a given $G_{0}, n_{d}$, and $T_{d}$, the PDR models gives us the value of column density or optical depth (at FUV wavelengths) at the disk edge. This specification acts as the inner boundary condition for equation (26).

We don't know (a priori) the density $n_{d}$ at the inner boundary, so we guess the value and invoke a constraint. By applying this constraint through an iterative procedure, we can converge on the correct value. The constraint that we invoke is that our solution must match onto the flow in the outer region beyond $r_{s}$. In this outer region, as outlined above, we assume a steady flow with $n \sim r^{-2}$ and hence the column density from $r_{s}$ to $\infty$ is given by $N_{s \infty}=n_{s} r_{s}$, or equivalently, $\tau_{s \infty}=\sigma_{F U V} n_{s} r_{s}$. Our solution to the differential equation defines when we get to the sonic point $\xi_{s}$, where the dust optical depth will have a calculated value $\tau_{F U V}\left(\xi_{s}\right)$. Thus, at the sonic point $\xi_{s}$, we know the value $\tau_{F U V}\left(\xi_{s}\right)$. But we also know the derivative $d \tau_{F U V} / d \xi$ and hence we know $n_{s}$ and also $\tau_{s \infty}$. In general, the value $\tau_{F U V}\left(\xi_{s}\right)$ will not be equal to the correct value $\tau_{s \infty}=\sigma_{F U V} n_{s} r_{s}$ $=\tau_{d} \xi_{s} g\left(\xi_{s}\right)$. In other words, we are searching for a zero of the function

$$
F_{\tau}\left(n_{d}\right)=\tau_{F U V}\left(\xi_{s}\right)-\tau_{d} \xi_{s} g\left(\xi_{s}\right),
$$

where $\xi_{s}$ is the sonic point defined previously and where $\tau_{F U V}\left(\xi_{s}\right)$ and $g\left(\xi_{s}\right)$ are the calculated solutions to the differential equations (25) and (26). If the function $F_{\tau}$ is not zero, then we can go back and choose a new estimate for $n_{d}$ (or $d \tau_{F U V} / d \xi$ at $\xi=1$ ) and integrate outwards again, and then repeat until we converge upon $F_{\tau}=0\left[\tau_{F U V}\left(\xi_{s}\right)=\tau_{s \infty}\right]$. In principle, we can carry out this iteration procedure for any value of the other unspecified constant $C$. Notice that the hydrostatic approximation is equivalent to assuming that $C=0$.

The value of $C$ specifies the mass outflow rate in that $\sqrt{C}$ is the dimensionless mass loss rate. In other words, by definition,

$$
\dot{M}=4 \pi r_{d}^{2} \mathcal{F}\langle\mu\rangle n_{d} a_{d} \sqrt{C} .
$$

But the mass outflow rate at the outer boundary (the sonic point) is given by

$$
\dot{M}=4 \pi r_{d}^{2} \mathcal{F}\langle\mu\rangle n_{d} a_{d} \xi_{s}^{2} g \sqrt{f} .
$$

Thus, for consistency, we must invoke the constraint $C=\xi^{4} g^{2} f$ at the sonic point. In other words, we are searching for the zero of the second function

$$
F_{\dot{M}}=C-\left[\xi^{4} g^{2} f\right]_{\xi_{s}} .
$$

One way to carry out the iteration procedure is as follows: First we estimate $C$. For that value of $C$, we estimate the density $n_{d}$. We then carry out the iteration procedure on $n_{d}$ until it has converged to the proper value (so that $F_{\tau}=0$ ) for the working value of $C$. In general, $C$ will not have the right value to conserve mass (to satisfy the second constraint $F_{\dot{M}}=0$ ), so we pick a new value of $C$. For the new value of $C$, we run the iteration procedure on $n_{d}$ until it converges, and so on. When convergence is reached, the value of the dimensionless constant $C$ determines the total effective mass outflow rate through equation (32). 


\subsection{Analytic Scalings}

The Appendix provides an analytic solution for the photoevaporative mass loss rate for the simple approximation that the external field $G_{0}$ heats the disk surface to a constant temperature $T_{s}$ to a critical depth $N_{C}$. Under this set of approximations, the solution for disks with $r_{d}<r_{g}$ takes the form

$$
\dot{M}=C_{0} N_{C}\langle\mu\rangle a_{s} r_{g}\left(\frac{r_{g}}{r_{d}}\right) \exp \left[-r_{g} / 2 r_{d}\right]
$$

where $C_{0}$ is a dimensionless constant of order unity and where $a_{s}$ is the sound speed appropriate for the temperature $T_{s}$. We can use our numerical results to provide a specification of the constant $C_{0}$ by matching the mass loss rates for a given value of $r_{d} / r_{g}$. This matching procedure is sensitive to the assumed matching point because the (numerically determined) temperature distribution does not suddenly drop at $N_{H}=N_{C}$, but rather continuously falls with increasing column density.

As shown in the Appendix, this scaling law for $\dot{M}$ does not drop appreciably with decreasing disk size $r_{d}$ until the disk is significantly smaller than the critical radius, i.e., until $r_{d} / r_{g} \lesssim 0.15$. The analytic treatment also shows that the mass loss rate from the disk surface (the vertical flow) is smaller than the mass loss rate from the disk edges (the radial flow) by a factor of $\sim\left(r_{d} / r_{g}\right)^{1 / 2}$. The flow from the disk edge thus dominates for $r_{d} \ll r_{g}$.

\subsection{Results}

The formulation developed thus far allows us to calculate the mass outflow rates from circumstellar disks, as a function of stellar mass $M_{*}$, outer disk radius $r_{d}$, temperature boundary conditions $T_{d}$, and the intensity $G_{0}$ of the external radiation field. The resulting fluid fields for a converged model are shown in Figure 3. In this system, a $30 \mathrm{AU}$ disk surrounds a $1.0 M_{\odot}$ star, and the star/disk system is exposed to an FUV radiation field of intensity $G_{0}=3000$. As shown in Figure 3, the flow begins subsonically at the disk edge (with Mach number $\mathcal{M} \sim 0.09$ ) and smoothly approaches the sound speed at a radius a few times larger than that of the disk. The flow speed and the temperature increase outwards, while the density decreases. All of the functions vary (nearly) as power-laws, as indicated by the nearly straight lines on the log-log plot. The density profile is actually a combination of power-law and exponential behavior (see eq. [35]). The power-law behavior is dominant for the regime of parameter space where substantial flow develops; when the exponential behavior dominates, the mass outflow rate becomes exponentially suppressed.

Figure 4 shows the mass loss rates as a function of disk radius for a typical disk surrounding a $M_{*}=1.0 M_{\odot}$ star embedded in external FUV radiation fields with $G_{0}=300-30,000$. For the central value $G_{0}=3000$, the figure shows the result for three different choices of the temperature at the inner disk edge, specifically $T(30 \mathrm{AU})=60 \mathrm{~K}, 75 \mathrm{~K}$, and $90 \mathrm{~K}$. The resulting evaporation

rates are relatively insensitive to this inner boundary condition and we will adopt the central value, $T(30 \mathrm{AU})=75 \mathrm{~K}$, as our working 'standard' value. Figure 4 shows that for a given FUV field 
(which roughly fixes $N_{C}$ and $a_{s}$ ) the mass loss rate decreases with shrinking disk radius $r_{d}$, as expected by the analytic scaling law (eq. [35]) where $\dot{M} \propto \exp \left[-r_{g} / 2 r_{d}\right]$. Our numerical approach contains approximations that do not allow us to find solutions for large $r_{d} / r_{g} \gtrsim 0.14$ (because the sonic point solution of eq. [30] becomes complex). Furthermore, the mass loss rate $\dot{M}$ drops rapidly with smaller $r_{d} / r_{g} \lesssim 0.14$ so that the gas is too dense (at the base) to be modeled accurately with the PDR code. Therefore, the numerical results are confined to a relatively small range of $r_{d} / r_{g}$, but a large range of $\dot{M}$. We can use our analytic approximation (eq. [35]) to provide estimates for the mass loss rates when $r_{d} / r_{g} \gtrsim 0.14$. The result is shown in Figure 5 for the case of $M_{\star}=1.0$ $M_{\odot}$ and $G_{0}=3000$, where we have specified the dimensionless constant $C_{0}$ to match the numerical solution.

Figure 4 also shows that the mass loss rates $\dot{M}$ are a sensitive function of the intensity $G_{0}$ of the FUV radiation field. For an external radiation field with $G_{0}=300$, the evaporation rate is almost inconsequential (for disks with $r_{d} \lesssim 100 \mathrm{AU}$ ). For stronger radiation fields with $G_{0}=$ $3000-30,000$, however, the mass loss rates are significant. The mass loss rates are sensitive to $G_{0}$ because higher values of the radiation intensity lead to higher temperatures and lower critical radii $r_{g}\left(\propto T^{-1}\right)$. Since $\dot{M} \propto \exp \left[-r_{g} / 2 r_{d}\right]$ (approximately), a modest increase in temperature can lead to a significant increase in the mass loss rate.

Figure 6 shows how the evaporation rate depends on the mass of the parental star. All of these models use an external FUV radiation field with $G_{0}=3000$ and assume our standard boundary conditions. The curves show the resulting mass loss rates for stellar masses $m=M_{*} /\left(1 M_{\odot}\right)=$ 0.25 - 1.0. Notice that the mass loss rate is a sensitive function of the central stellar mass $(\mathrm{m})$; at $r_{d} \approx 20 \mathrm{AU}$, the evaporation rate varies by an order of magnitude over the range of stellar masses used here. The mass loss rates are sensitive to $M_{*}$ because the critical radius $r_{g} \propto M_{*}$. Since $\dot{M} \propto \exp \left[-r_{g} / 2 r_{d}\right]$, decreasing the stellar mass (with a corresponding decrease in $r_{g}$ ) leads to a rapidly increasing mass loss rate $\dot{M}$. As we explore in greater detail below $(\S 7)$, this result implies that low mass stars can easily lose the gas in their circumstellar disks.

Figure 7 shows the various length scales in the problem. Here, the disk scale height $H_{d}$, the sonic radius $r_{s}$, and the critical radius $r_{g}$ are plotted as a function of disk radius $r_{d}$. For this model, the stellar mass $M_{*}=1.0 M_{\odot}$ and the FUV radiation field has intensity $G_{0}=3000$. The critical radius $r_{g}$ depends on the gas temperature according to equation (1). In these models, the temperature approaches a constant value in the outer region where the flow is supersonic. For systems with larger disk radii $r_{d}$, the flow reaches supersonic speeds more easily, at lower temperature, and the critical radius $\left(r_{g} \propto T_{s}^{-1}\right.$ as defined here) increases with $r_{d}$. The curves depicting both the sonic point and the critical radius show (non-monotonic) structure, which is a reflection of the structure in the relationship between temperature and visual extinction (Fig. 2). As expected, the critical radius is always much larger than the disk radius, by almost an order of magnitude, for this regime of parameter space. The critical radius $r_{g} \approx 4 r_{s}$ for systems with relatively high $\dot{M}$ as shown here (where the factor $(1-8 f / \beta)^{1 / 2} \ll 1$ in eq. [30]). For smaller mass loss rates (smaller disk radii $r_{d}$ ), $r_{g} \approx 2 r_{s}$ as noted in $\S 4$.4. Another measure of how far the disks 
are from being supercritical is to compare the minimum value of $r_{g}$ (corresponding to the highest temperature accessible for a given radiation field) with the disk size $r_{d}$. This minimum $r_{g}$ is about $160 \mathrm{AU}$ for the case shown here. Nonetheless, as shown in the previous figures, the disk experiences significant mass loss in this subcritical state. Notice also that the length scales obey the ordering of equation (10).

\subsection{Evaporation Time Scales}

To convert our results into time scales for disk evaporation, we need to account for the mass supply in the disk. Here we assume that the surface density is given by the simple power-law form

$$
\Sigma(r)=\Sigma_{0}\left(\frac{r_{0}}{r}\right)^{p}
$$

where $r_{0}$ is the initial outer radius of the disk and $\Sigma_{0}$ is the corresponding outer surface density. The coefficient $\Sigma_{0}$ is determined by the total starting disk mass, i.e.,

$$
M_{d 0}=\frac{2 \pi}{2-p} \Sigma_{0} r_{0}^{2},
$$

where we have made the approximation $r_{0} \gg R_{*}$ (the stellar radius, or inner disk radius). As the disk evaporates, we assume that mass loss occurs from the outside to the inside; specifically, we assume that all of the mass is evaporated from a given annulus before the mass loss moves inward. The disk mass as a function of time is then given by

$$
M_{d}\left(r_{d}\right)=M_{d 0}\left(\frac{r_{d}}{r_{0}}\right)^{2-p}
$$

where $r_{d}$ is the time-dependent disk radius $\left(r_{d}<r_{0}\right)$. For the sake of definiteness, we take $p=3 / 2$ throughout this paper, and normalize the surface density such that

$$
M_{d}\left(r_{d}\right)=0.05 M_{*}\left(\frac{r_{d}}{30 \mathrm{AU}}\right)^{1 / 2}
$$

Notice that this formula remains valid for disk radii $r_{d}>30 \mathrm{AU}$. The evaporation time $t_{\text {evap }}$, for a given disk radius, is thus given by

$$
t_{\text {evap }}=\frac{M_{d}\left(r_{d}\right)}{\dot{M}\left(r_{d}\right)}
$$

\subsection{Coupling of Photoevaporation and Disk Accretion}

In addition to photoevaporation from its outer edges, the disk will also experience disk accretion as long as it has an internal source of viscosity. Since the disk is finite, material cannot move inwards at all radial locations. In particular, the outer disk edge will expand outwards on the diffusion time scale given by

$$
\tau_{d i f f}=r_{d}^{2} / \nu_{d}
$$


where $\nu_{d}$ is the viscosity. The evaporation time scale decreases with disk radius, whereas the disk diffusion time increases with disk radius. In other words, as photoevaporation takes place and the disk shrinks, the time scale required for photoevaporation grows longer, but the time scale for the disk to replenish the mass supply (through accretion and spreading) grows shorter. The disk will thus obtain a quasi-equilibrium state in which the time scales for photoevaporation and disk accretion are in balance. The disk will thus maintain a fixed radius for as long as both processes are effective.

We can estimate the disk radius at which photoevaporation and disk accretion are balanced. The evaporation time scales are the main focus of this paper. The diffusion time scale is given by equation (41), where the viscosity can be written in terms of an 'alpha prescription' via

$$
\nu_{d}=\frac{2}{3} \alpha a_{d} H_{d}
$$

where $\alpha$ is the usual viscosity parameter and where we evaluate the sound speed and scale height at the outer disk edge. In Figure 8 we show the resulting disk accretion time scales along with the photoevaporation time scales calculated in this paper. The results are shown for a star with mass $M_{*}=1.0 M_{\odot}$ with a disk exposed to an FUV radiation field with $G_{0}=3000$. We assume that the disk mass $M_{d}=0.05 M_{\odot}\left(r_{d} / 30 \mathrm{AU}\right)^{1 / 2}$. Although the photoevaporation time scale is relatively insensitive to the disk temperature at the outer edge (which represents the inner boundary condition to the outflow problem), the disk accretion time is more sensitive. Figure 8 shows the results for three choices of temperature scale, $T_{d}\left(r_{d}=30 \mathrm{AU}\right)$, as labeled.

Disks are expected to form with a radius $r_{d} \sim 100 \mathrm{AU}$, somewhat larger than the crossover radii shown in Figure 8. In the long term, the disk radius will shrink down to the size at which photoevaporation and disk spreading (from accretion) are in balance. This state should be an equilibrium: If the disk radius were to grow, photoevaporation would win over disk spreading and the disk would decrease its size. If the disk became too small, then the photoevaporation would become much less effective but disk accretion would replenish the material in the vacated region. The disk will thus maintain this equilibrium size as viscosity drains material onto the star and photoevaporation drains material outward into the interstellar medium. This balance will continue until the disk surface density becomes so small that angular momentum transport (accretion) is no longer effective (see also Clarke et al. 2001, Matsuyama et al. 2003ab).

\section{GAS REMOVAL FROM THE EARLY SOLAR NEBULA}

The previous sections provide a working formulation to calculate evaporation rates from circumstellar disks embedded in external FUV radiation fields. Our first application of these results is to our own solar system. Here, the planets Neptune and Uranus are seriously depleted in hydrogen gas compared to solar abundances. On the other hand, both Jupiter and Saturn are relatively gas rich. If the solar system formed in a group or cluster environment, which in turn provides a strong 
external radiation field, then gas would be lost from the outer solar nebula through the mechanism developed above.

To fix ideas, we first assume that the disk starts off with outer radius $r_{i}=r_{g}$. Given that $r_{g}$ has a typical size of $100 \mathrm{AU}$ (for the temperatures produced by a cluster radiation field), this assumption is quite reasonable. The initial mass loss rate is then given by equation (9), the mass loss rate for a supercritical disk. The starting time scale $t_{i}$ for the disk to change its mass content is thus given by

$$
t_{i}=\frac{M_{d i}}{\dot{M}_{i}}=\frac{a_{S} \sigma_{F U V} M_{d i}}{4 \pi\langle\mu\rangle G M_{*}} .
$$

For a temperature of $T=1000 \mathrm{~K}$, the fiducial mass loss rate is about $\dot{M}_{i} \approx 10^{-7} M_{\odot} \mathrm{yr}^{-1}$. For a relatively large starting disk mass of $M_{d i}=0.1 M_{\odot}$, e.g., the corresponding time scale is $t_{i} \approx 1$ Myr. As a result, if our initial solar nebula extended out to the escape radius near $100 \mathrm{AU}$ (and if our solar system formed within a respectably large birth aggregate so that $T \sim 1000 \mathrm{~K}$ ), then the nebula would evaporate relatively quickly (at least at first) and become smaller.

Specifically, the nebula would shrink until its outer radius $r_{d}$ became significantly smaller than $100 \mathrm{AU}$. As a result, the solar nebula would rapidly attain a smaller radius of $r_{d} \sim 30 \mathrm{AU}$, a smaller mass of $M_{d} \sim 0.05 M_{\odot}$. With these properties, the nebula would be within the subcritical regime and it would then continue to evaporate as described in $\S 4$. Notice that as the solar nebula shrinks, the evaporation time scale is affected by two competing effects: As $r_{d}$ decreases, the mass loss rate gets smaller, which would tend to increase the evaporation time scale. However, the disk mass decreases also, and this effect compensates to some degree. In addition, the outer disk edge spreads outward on the viscous diffusion time scale; if the disk has enough viscosity, then the nebula would maintain a quasi-equilibrium size as it evaporates (see Figure 8).

The evaporation time scales for the solar nebula (a disk surrounding a $1.0 M_{\odot}$ star) are shown in Figure 9, as a function of disk radius, for three different intensities of the external FUV radiation field. As a benchmark, a $30 \mathrm{AU}$ disk with mass $M_{d}=0.05 M_{\odot}$ embedded in a radiation field with $G_{0}=3000$ has an estimated evaporation time scale of $14 \mathrm{Myr}$, which is somewhat larger than the fiducial time scale of $10 \mathrm{Myr}$ required for giant planet formation. If the starting disk mass had the much lower value $M_{d}=0.01 M_{\odot}$, the evaporation time would be only about $3 \mathrm{Myr}$ and the $G_{0}=$ 3000 radiation field could substantially affect planet formation. For a (much larger) FUV radiation intensity of $G_{0}=30,000$ (typical of large clusters like the Trapezium), the evaporation time scale for a $30 \mathrm{AU}$ disk (again with $M_{d}=0.05 M_{\odot}$ ) is only about $4 \mathrm{Myr}$, comfortably less than the time scale expected for giant planet formation. This level of radiation is able to evaporate the outer portion of the early solar nebula and could provide an explanation for the observed deficit of gas in the ice giants (Neptune and Uranus). Even for this radiation field, however, the time scale for evaporation is about $20 \mathrm{Myr}$ at 10 AU (the location of Saturn's current orbit) and much longer at $5 \mathrm{AU}$ (the location of Jupiter). We conclude that the region of the solar nebula where Jupiter and Saturn reside are relatively safe from photoevaporation over the time scales relevant for giant planet formation. 
Substantial mass loss from the ice giant region of the early solar nebula thus requires an intense FUV radiation field with $G_{0} \approx 30,000$. What type of solar birth environment is expected to produce such a radiation field? For a benchmark distance of $r=0.2 \mathrm{pc}$, for example, this radiation level can be provided by a single $30 M_{\odot}$ star. Such large stars are exceedingly rare - only about 1 out of 2400 stars are at least this massive, according to the standard form of the stellar initial mass function $\left(f=d N_{*} / d M_{*} \sim M_{*}^{-2.35}\right.$; Salpeter 1955), so a large cluster like the Trapezium is generally needed. Nonetheless, stars of similar mass have been invoked to provide enrichment of short-lived radiative species in the early solar nebula (e.g., Cameron et al. 1995), although the preferred distance from the massive star is much larger for optimal enrichment and solar system survival (e.g., see Boss \& Foster 1998).

In such an energetic environment, the EUV radiation fields will be substantial and can affect the process of planet formation, in addition to the FUV radiation considered here (e.g., SH99, Armitage 2000, Adams \& Laughlin 2001, Adams \& Myers 2001). However, the effect of the EUV field in such energetic environments is somewhat subtle. Even in the presence of strong EUV fields, the FUV fields can dominate the mass loss rate by creating a neutral flow from the disk surface which absorbs the EUV flux at an ionization front that lies at several disk radii, far out into the supersonic region of the neutral flow. Störzer \& Hollenbach (1999) showed that for $r_{d}>r_{g}$ and Trapezium-like conditions, the FUV dominates the mass loss rate for $r_{d} \sim 100 \mathrm{AU}$ disks at distances of $d \approx 0.02$ pc to $0.4 \mathrm{pc}$ (where $G_{0}=10^{4}-10^{6}$ ). SH99 did not consider the case where $r_{d}<r_{g}$. Once the disk shrinks to $r_{d} \lesssim 0.2 r_{g}$, however, the neutral mass loss rate will decline, the EUV flux will penetrate to the disk surface, and the EUV mass loss rate will take over. We have calculated the critical disk size $r_{\text {cr }}$ at which EUV begins to dominate $\dot{M}$ for flux from a Trapezium-like $\Theta^{1}$ Ori $\mathrm{C}$ at distances such that $G_{0}=30,000$. We find that FUV radiation drives the mass loss until the disk radius shrinks to sizes less than about $r_{d} \lesssim 10 \mathrm{AU}$, at which point EUV takes over. The EUV flux evaporates the disk from $10 \mathrm{AU}$ down to $2 \mathrm{AU}$ in about $30 \mathrm{Myr}$. The evaporation time scale grows rapidly if the disk shrinks below $r_{d} \lesssim 0.2 r_{g} \approx 2$ AU (where $r_{g} \sim 10$ AU for EUV heating, which gives $T \approx 10^{4} \mathrm{~K}$ ). Beyond this point, not even EUV photons can lift the gas out of the potential well. We have also calculated the critical disk size for conditions in a moderate sized cluster (where $N_{\star} \sim 300$ so that the largest star $M_{*} \sim 9 M_{\odot}$ ) at distances such that $G_{0} \approx 3000$. With a lower mass star as the power source, the ratio of EUV to FUV photons is lower. Here, the FUV dominates again, until the disk radius $r_{d} \lesssim 10 \mathrm{AU}$, where the mass loss rates are very small $\left(\dot{M} \sim 10^{-11}-10^{-10} M_{\odot} \mathrm{yr}^{-1}\right)$ and the evaporation time scales are extremely long $(t \gg 100 \mathrm{Myr})$.

To summarize this section, we find that an FUV radiation field of $G_{0}=30,000$ can potentially explain the deficit of gas in the ice giants in our solar system. This level of radiation can effectively evaporate the gas in the outer portion of the early solar nebula where Neptune and Uranus now reside. The $\sim 15$ Earth mass cores that form there would have little gas to accrete and could thus develop into ice giants (as observed). These same radiation levels will leave the remainder of the solar nebula intact, with sufficient gas for giant planet formation in the Jupiter/Saturn region of the nebula. In general, however, we expect external radiation fields of this required intensity to be 
somewhat rare. Unfortunately, a general assessment of the probability for forming solar systems to experience such radiation levels remains an open issue, which must be left for future work. We also note that recent work suggests that the ice giants may have formed at smaller orbital radii and then migrated outwards through scattering encounters with Jupiter (e.g., Thommes, Duncan, \& Levison 1999). In this event, the mass loss mechanism considered here would allow the ice giants to remain impoverished in gas even after they migrate.

\section{FORMATION OF THE KUIPER BELT AND DEBRIS DISKS}

The Kuiper Belt and the question of its formation is an interesting astronomical issue for several reasons. First, since the Kuiper Belt is an important part of our solar system, any complete theory of solar system formation must account for its origin. Second, the existence and observed structure of the Kuiper belt can be used to place constraints on the process of solar system formation, including properties of the solar birth environment (e.g., Adams \& Laughlin 2001). Third, debris disks are observed around many nearby stars (e.g., Backman, Gillett, \& Witteborn 1992) and the dust in these systems is provided by shattering collisions between planetesimals, which (apparently) orbit about the central stars at distances $r=10-200$ AU. In other words, debris disks contain rocky bodies that are roughly analogous to the Kuiper Belt objects in our solar system. In addition, at a given age, the amount of dust orbiting stars of similar spectral type shows great variation. Some young stars show no evidence for debris disks, whereas some older stars are accompanied by copious amounts of debris dust. In this section, we show how photoevaporation can affect the formation of the Kuiper Belt, in our solar system and others.

Specifically, the inventory of Kuiper Belt objects and their associated debris dust will be suppressed if the primordial dust is removed along with gas - via photoevaporation - during the first million years in the life of the star/disk system (before dust has had a chance to coagulate to significant sizes $b \sim 1 \mathrm{~cm}$ ). To study these effects, we need to estimate the critical size required for dust grains to become entrained in the outflowing gas and the coagulation time scale required for grains to attain that critical size. The relative importance of photoevaporation then depends on the ratio of the coagulation time scale (calculated below) to the evaporation time scale (as calculated in $\S 4$ ). Variations in this ratio will lead to variations in the abundance of Kuiper belt objects and debris dust later on.

The photoevaporation times vary widely and depend sensitively on the mass and proximity of the nearest massive star. In one limit, a star could be born within a large cluster like the Trapezium (e.g., Hillenbrand \& Hartmann 1998; Störzer \& Hollenbach 1999), where its outer disk $(r \gtrsim 50-100$ AU) evaporates much faster than dust can coagulate. Later in their lives, such stars would show little or no evidence for extended debris dust because the outer Kuiper Belt objects could never form. In the other extreme, a star born in isolation can produce numerous Kuiper Belt objects out to large distances from the central star, and will exhibit extended debris dust long after its formation. Most stars form in environments between these two extremes. 


\subsection{Critical Size for Dust Entrainment During Photoevaporation}

In order for a dust particle to be entrained in flow and carried off as the gas evaporates, two conditions must be met: (i) The drag force of the gas moving past the dust particle must be greater than the gravitational force on the dust particle from the central star. (ii) The force must act over a sufficient time, long enough to enable the dust particle to reach the escape speed from the system. For gas flowing at the escape speed, the first condition can be written (approximately) as

$$
b<\frac{3 \Sigma_{g a s}}{4 \rho_{g r}}\left(\frac{r}{2 H}\right)
$$

where $b$ is the radius of the dust particle, $\Sigma_{\text {gas }}$ is the gas surface density of the disk at radius $r, H$ is the scale height of the gas at $r$, and $\rho_{g r}$ is the mass density of the grain material. The second condition is roughly equivalent to the requirement that the dust particle must encounter its own mass in gas molecules (moving at the escape speed $v_{e s c}$ ) for the dust grain to be accelerated to $v_{e s c}$. If a typical dust particle initially resides halfway (in column density) between the midplane and the disk surface, then a gas mass column $0.25 \Sigma_{\text {gas }}$ will sweep by the dust particle. This second condition can be written in the form

$$
b<3 \Sigma_{g a s} /\left(16 \rho_{g r}\right) .
$$

This second condition is the more stringent because $r / 2 H>1$. As a result, some relatively large particles can meet the first condition and initially move outward from the star, but the gas flow past them dwindles before they reach escape velocity and they fall back into orbit (unless they also meet the second condition).

Next we assume that the newly formed disk has a gas surface density of the form given by

equation (36) with $p=3 / 2$. If the coagulated grain material has a density $\rho_{g r} \sim 1 \mathrm{~g} \mathrm{~cm}^{-3}$, the second condition (eq. [45]) can be evaluated to obtain the limit

$$
b<0.13 \mathrm{~cm}\left(\frac{M_{d}}{0.01 M_{\odot}}\right)\left(\frac{r}{100 \mathrm{AU}}\right)^{-1 / 2}\left(\frac{r_{d}}{100 \mathrm{AU}}\right)^{-3 / 2}
$$

where $r$ is the radius at which we evaluate the limit and $r_{d}$ is the location of the outer disk edge. If we are concerned with dust entrainment in the region $30 \mathrm{AU}<r<100 \mathrm{AU}$, where Kuiper Belt objects ultimately formed in our Solar System (and if $M_{d} \sim 0.01 M_{\odot}$ and $r_{d} \sim 100 \mathrm{AU}$ ), then the critical size for dust coagulation is $b \approx 0.1-1 \mathrm{~cm}$. Once dust particles coagulate to larger sizes, they will remain bound to the system as the gas evaporates. These remaining rocks can eventually form Kuiper Belt objects and debris dust.

\subsection{Coagulation Timescales}

A standard scenario for dust coagulation in protostellar disks has been developed (e.g., Weidenschilling 1997). During the collapse of a molecular cloud core, gas and dust hit the forming 
circumstellar disk in freefall, with typical speeds of $\sim 3 \mathrm{~km} \mathrm{~s}^{-1}$ at $100 \mathrm{AU}$. The dust passes through an accretion shock and comes to rest with the gas in the upper atmosphere of the disk. At this stage, the dust particles are mostly interstellar in size, with radii $b \lesssim 0.1 \mu \mathrm{m}$. Within the disk, the dust has a tendency to settle to the midplane (due to the vertical component of the stellar gravity force). The gas is supported by gas pressure, but the dust grains settle slowly through the gas, at settling speed $v_{\text {set }}$, resisted by the drag force exerted by collisions with gas molecules. For the small particles, the thermal speed $a \sim 0.1 \mathrm{~cm} \mathrm{~s}^{-1}$ is larger than the settling speed $v_{\text {set }}$, and the dust particles remain in the upper atmosphere until they begin to collide and coagulate.

A dust particle grows until its settling speed $v_{\text {set }}$ exceeds the sound speed $a$, and the particle then begins its descent toward the midplane. As it moves through gas and smaller dust particles, the dust grain sweeps up smaller grains and grows larger. The dust particle falls at its terminal speed, with the force of gravity balanced by the gas drag. As the dust grain grows, its terminal speed increases, so that the dust particle accelerates at first. When the dust approaches the midplane, however, the vertical component of the stellar gravity diminishes, and the terminal speed decreases. As a rough estimate, the dust particle attains settling speeds $v_{\text {set }} \sim 100 \mathrm{~cm} \mathrm{~s}^{-1}$ at $100 \mathrm{AU}$ and grows to sizes of $b \sim 1 \mathrm{~mm}$ (for disk mass $M_{d} \sim 0.01 M_{\odot}$ ) by the time it reaches the midplane. The descent to the midplane takes about $300-1000$ Keplerian orbits around the star, largely independent of the disk surface density. This result is due to the cancellation of two opposing factors. At a given drift speed, a higher density disk leads to greater gas drag, which slows down the particles. However, a higher density disk also has more small dust particles that are available for coagulation, so the particle grows faster. The increased gravitational force offsets the increased gas drag. The precise number of Keplerian orbits required depends on the fluffiness (or fractal properties) of the coagulating dust particle as it descends. As a working estimate, we assume that the descent to the disk midplane corresponds to about 300 Keplerian orbits (see Weidenschilling 1997). In this scenario, the settling time controls the rate of coagulation. The time scale for small dust particles to thermally coagulate in the upper atmosphere is almost always shorter than the settling time of the somewhat larger particles that make the descent. The coagulation time scale is thus given by

$$
t_{\text {coag }} \simeq 3 \times 10^{5} \mathrm{yr}\left(M_{*} / 1 \mathrm{M}_{\odot}\right)^{-1 / 2}(r / 100 \mathrm{AU})^{3 / 2} .
$$

For the radii of interest for Kuiper belt formation, roughly 30 - $100 \mathrm{AU}$, the coagulation time scale is $t_{\text {coag }} \approx 0.05-0.30 \mathrm{Myr}$.

\subsection{Comparison of Evaporation and Coagulation Time Scales}

The previous subsection argues that the dust coagulation time scale is typically less than 1 Myr for radial locations corresponding to the present-day Kuiper belt. In order for the evaporation time scale to compete with this (short) coagulation time scale, the photoevaporation time scale must lie in the supercritical regime. In this case, the time required for photoevaporation can be 
written

$$
t_{\text {evap }} \approx \frac{M_{d} \sigma_{F U V}}{4 \pi \mathcal{F}\langle\mu\rangle a_{S} r_{d}} \approx 0.6 \mathrm{Myr} \mathcal{F}^{-1}\left(\frac{a_{S}}{1 \mathrm{~km} / \mathrm{s}}\right)^{-1}\left(\frac{r_{d}}{100 \mathrm{AU}}\right)^{-1}\left(\frac{M_{d}}{0.03 M_{\odot}}\right)
$$

where we have scaled the result compared to typical parameter values. Equating the coagulation time scale (eq. [47]) with the evaporation time scale (eq. [48]), we find the constraint required for Kuiper belt formation to be compromised, i.e.,

$$
\left(\frac{a_{S}}{1 \mathrm{~km} / \mathrm{s}}\right)\left(\frac{r_{d}}{100 \mathrm{AU}}\right)^{5 / 2} \geq 2 \mathcal{F}^{-1}\left(\frac{M_{d}}{0.03 M_{\odot}}\right)\left(\frac{M_{*}}{1.0 M_{\odot}}\right)^{1 / 2} \approx 3
$$

where the approximate equality (on the right hand side of the inequality) applies for the minimum mass solar nebula. Even at the (rather large) radius of $100 \mathrm{AU}$, a sound speed of $3 \mathrm{~km} / \mathrm{s}$ (corresponding to a temperature of $\sim 1360 \mathrm{~K}$ ) is necessary to evaporate the disk faster than dust grains can coagulate. For the highest intensity FUV radiation field considered in this paper, $G_{0}=30,000$, the temperature at the $A_{V}=1$ surface is only about $600-700 \mathrm{~K}$. In other words, the coagulation time remains shorter than the evaporation time for nearly all of the expected radiation fields that young solar systems might be exposed to.

Alternately, for a given radiation field and hence a given estimate for the sound speed at the sonic point, one can derive a cutoff radius for the existence of dust (by solving equation [49] for the radius). For a radiation field of $G_{0}=3000$, e.g., the PDR models indicate that the sound speed $a_{S} \approx 1.4-2.2 \mathrm{~km} / \mathrm{s}$ and hence the cutoff radius is predicted to lie in the range $r_{c} \approx 110-140 \mathrm{AU} ;$ for a radiation field with $G_{0}=30,000, a_{S} \approx 1.5-3.3 \mathrm{~km} / \mathrm{s}$ and the cutoff radius $r_{c} \approx 96-130$ AU. At radial locations inside the cutoff radius, the coagulation time is shorter than the evaporation time and dust successfully transforms itself into centimeter-sized rocks (essentially gravel). Outside $r_{c}$, most of the material (both gas and dust) is carried off to the interstellar medium. These considerations thus predict a reasonable sharp cutoff for Kuiper Belt objects in circular orbits ${ }^{2}$ beyond the radius $r_{c}$. Notice that this cutoff radius is safely beyond the observed outer "edge" of our Kuiper belt at 50 AU (see, e.g., Allen, Bernstein, \& Malhotra 2002, Trujillo \& Brown 2001).

Particle coagulation proceeds to the critical size before the gas and small dust evaporates at $r \lesssim 100$ AU, even under extreme (e.g., Trapezium-like) conditions. Although the proplyds in Orion have shrunk to $r_{d} \lesssim 30 \mathrm{AU}$ in $\sim 0.3 \mathrm{Myr}$ (e.g., SH99), this coagulation model predicts that a significant fraction of the dust will have quickly coagulated into particles with $b \gtrsim 1 \mathrm{~cm}$. These large dust grains (rocks) will still reside in the disk, from the current disk radius of $r_{d} \lesssim 30 \mathrm{AU}$ (the boundary for gas and small dust particles) out to about 100 AU. These rocky bodies have little optical opacity, but are available to form Kuiper Belt objects. Although definitive models of planet formation are not yet available, the accumulation of these dust grains into planetesimals (and planets) must proceed differently in the outer disk (with no gas) and the inner disk (within $r \sim 30$ AU where gas is retained much longer).

\footnotetext{
${ }^{2}$ We emphasize circular orbits because Kuiper Belt objects formed at smaller radii can attain highly eccentric orbits with large semi-major axes through scattering interactions with giant planets (and smaller bodies).
} 
We conclude this section with two important caveats, which could significantly change the estimated radius $r_{c}$, as set by the intersection of the coagulation time with the evaporation time. First, the evaporation time scale is proportional to the disk mass, whereas the coagulation time scale is almost independent of the disk mass. As a result, disks with lower initial masses will have lower crossover radii. Second, the settling time scale depends on the fluffiness of the coagulating particles and this fluffiness remains uncertain. More porous grains will settle more slowly and the cutoff radius $r_{c}$ will decrease accordingly. As a result, we cannot completely rule out the idea that photoevaporation of the solar nebula caused the observed sharp cutoff in our Kuiper belt at 50 AU (if the Sun formed in the high radiation environment of a large cluster).

\section{SUPPRESSION OF PLANET FORMATION IN CLUSTERS}

The photoevaporation mechanism explored in this paper not only affects the (possible) loss of gas from our own solar nebula, but also implies that planet formation can be suppressed in other systems. In particular, circumstellar disks associated with solar type stars can be readily evaporated in sufficiently large clusters, whereas disks around smaller (M type) stars can be evaporated in more common, smaller groups.

As an observational example of a 'large' cluster, consider the Hyades, a relatively nearby stellar aggregate that is being searched for planets. At the present time, the cluster mass is estimated to lie in the range $M_{C}=300-460 M_{\odot}$ (e.g., Perryman et al. 1998). The metallicity is relatively high, $[\mathrm{Fe} / \mathrm{H}]=0.14 \pm 0.05$. Given the apparent correlation of extra-solar giant planets with metallicity (e.g., Gonzales et al. 2001), we would expect the Hyades stars to readily form giant planets in the absence of any disruption effects from the background cluster.

To assess the effects of the cluster environment on planet formation in the Hyades, we need to estimate its properties during its first $10 \mathrm{Myr}$ of evolution when planets are expected to form. The Hyades now has an age of $\sim 700 \mathrm{Myr}$, so we must extrapolate back to its youth. This transformation has been done (Kroupa 1995) and indicates that the cluster had a mass $M \sim 1300 M_{\odot}$ and $N_{\star} \sim$ 3000 at its dynamical beginning. These cluster properties are roughly comparable to those of the Trapezium cluster today (Hillenbrand \& Hartmann 1999), where circumstellar disks are observed to be actively evaporating (see McCaughrean \& O'Dell 1996 and many others). In addition, we note that $N_{\star} \sim 3000$ is the number of stars left in the system after gas removal from the young cluster. During its first few Myr of life, the cluster must contain even more stars $N_{0}=N_{\star} / \mathcal{F}_{\star}(\epsilon)$. The fraction $\mathcal{F}_{\star}$ of stars remaining after gas removal depends on the star formation efficiency of the cluster, but is expected to be $\mathcal{F}_{\star} \sim 3 / 4$ (see Adams 2000 for further detail). The formative stage of the Hyades could thus have nearly $N_{\star} \sim 4000$ stellar members. In estimating the radiation field of the cluster during its first $10 \mathrm{Myr}$ of life, we need to find the expected number of $\mathrm{O}$ and B stars in a randomly selected population of $N_{\star} \sim 4000$ (where we assume that the massive stars tend to form in the cluster center, as observed, so that they are not likely to leave the cluster during the gas removal adjustment phase). For a standard stellar IMF, a collection of 4000 stars 
should contain $\sim 12-16$ stars with $M_{*}>8 M_{\odot}$ (large enough to explode as supernovae) and should produce enough ultraviolet radiation to effectively evaporate circumstellar disks on a short time scale.

Such large clusters can readily produce FUV radiation fields with $G_{0}=30,000$, strong enough to affect planet formation. As shown in Figure 9 for solar type stars, the disk evaporation time scale is comparable to the expected planet formation time scale $(10 \mathrm{Myr})$ for disk radii $r_{d} \approx 15$ $\mathrm{AU}$ (note that the disk accretion time scale is also $\sim 10 \mathrm{Myr}$ for $r_{d}=15 \mathrm{AU}$ and $\alpha=10^{-4}$; see Figure 8 and equation [41]). Giant planet formation could thus be inhibited in large clusters like the Hyades. In addition, even though such planets could still form in the disk region $r=5-15$ AU, little disk mass (outside that region) would be available to drive planet migration. Thus, FUV radiation fields can alter the expected numbers and locations of planets for solar systems forming in large clusters, and may account for the observed underabundance (so far) of giant planets orbiting close to their central stars in the Hyades (e.g., Paulson et al. 2004, Cochran, Hatzes, \& Paulson 2003, Hatzes \& Cochran 2000). A similar deficit of planets has been found in the globular cluster 47 Tucanae (Gilliland et al. 2000), while populous metal-rich open clusters such as NGC 6791 are currently being surveyed (Mochejska et al. 2002). We note that if giant planets form rapidly through gravitational instability (e.g., Boss 2000), then we would not expect an anti-correlation of giant planets with the strength of the radiation fields.

The effect of FUV radiation on planet formation is more dramatic for stars of lower mass. Figure 10 shows the evaporation time scale for circumstellar disks exposed to a moderate FUV radiation field with $G_{0}=3000$. The evaporation time decreases rapidly with the mass of the central star, due to its (weaker) gravitational binding energy. Stars with $M_{*}=0.25 M_{\odot}$, which lie near the peak of the stellar mass distribution, will evaporate down to disk radii of 7 AU during the 10 Myr time interval of planet formation. We thus anticipate that giant planet formation can be seriously inhibited around low mass stars. However, these considerations do not preclude the formation of rocky terrestrial planets. The discussion of the previous section indicates that dust grains can easily coagulate on sufficiently short time scales to avoid being removed via photoevaporation. If stars form in reasonably large ensembles, then photoevaporation should remove gas, but not rocky dust grains, from the mass reservoir available to form planets. This effect is strongest for the least massive stars, so a clean prediction emerges: The metallicity of planets should increase with decreasing mass of the parental stars (for a given stellar metallicity). The magnitude of this trend depends on the typical intensity of radiation fields in star forming regions, and these radiation fields, in turn, depend on the size and density of those regions.

\section{SUMMARY AND DISCUSSION}

In this paper, we have studied the photoevaporation of small circumstellar disks $\left(r_{d}<r_{g} \sim 100\right.$ $\mathrm{AU})$ due to the heating by FUV radiation from the stellar birth environment. Because this work

applies to small disk radii, we can determine the effects of photoevaporation on inhibiting planet or 
planetesimal formation in the disk region where $r=10-100$ AU. This work complements previous studies, which have considered the evaporation of circumstellar disks due to EUV radiation from their parental stars (e.g., Shu et al. 1993) and the evaporation of large disks $\left(r_{d} \gtrsim 100 \mathrm{AU}\right)$ due to UV radiation in large clusters like the Trapezium (e.g., SH99). We show that FUV photoevaporation is likely to dominate EUV evaporation both in large clusters (e.g., $N_{\star} \approx 4000, G_{0} \approx 30,000$ ) and in more moderate sized groups (e.g., $N_{\star} \approx 300, G_{0} \approx 3000$ ), until the disks shrink to sizes $r_{d} \lesssim 10$ AU. By the time disks evaporate to such small radii (on time scales $t \gtrsim 30 \mathrm{Myr}$ ), the major episodes of planet formation are expected to be over, so that EUV photoevaporation does not generally play an important role in affecting planet formation.

[1] For solar type stars, with $M_{*} \approx 1 M_{\odot}$, relatively intense FUV radiation fields are required for significant photoevaporation to take place. In particular, FUV radiation with $G_{0}=30,000$ will efficiently evaporate disks with radii down to $r_{d} \sim 20 \mathrm{AU}$ on time scales of $\sim 10 \mathrm{Myr}$. The outer parts of these circumstellar disks can be effectively evaporated through the action of this level of FUV radiation, which is expected to be present in the cores of dense stellar clusters (e.g., $d \lesssim 0.7$ pc with $N_{\star} \approx 4000$ ).

[2] In our own solar system, the relative paucity of gas in Neptune and Uranus can be understood if the outer solar nebula $(r \gtrsim 20 \mathrm{AU})$ is stripped of its gas before the planets complete their formation ( $(5)$. The action of FUV radiation can remove enough gas on a sufficiently rapid time scale if the early solar system is exposed to FUV radiation fields with intensity $G_{0} \geq 30,000$. We expect such strong FUV radiation fields to be somewhat rare.

[3] FUV radiation fields can affect the formation of Kuiper belt objects and other rocky bodies in the outer portion of our solar system, and others. In these systems, dust grains coagulate as they settle and eventually grow too large to be removed from the disks. This process competes against evaporation, which acts to remove gas and dust from the disk. We find that dust coagulation tends to take place more rapidly (than mass loss) for radii less than a cutoff radius $r_{c} \approx 100 \mathrm{AU}$, even in relatively harsh stellar birth environments (§6). As a result, Kuiper belt objects, and the debris dust that they generate later on, can be formed (out to $r \sim 100 \mathrm{AU}$ ) around most stars. However, we cannot completely rule out the possibility that photoevaporation in the solar nebula could have produced the observed cutoff in Kuiper Belt objects at $r_{c} \sim 50$ AU.

[4] Relatively large clusters contain B stars (and even O stars) with high probability. Sufficiently rich clusters thus provide a hostile environment for giant planet formation because the FUV radiation from the background cluster is effective at removing gas from nebular disks. Applying this result to known clusters, such as the Hyades $(\S 7)$, we find that giant planet formation can be compromised in such environments.

[5] We have calculated (numerically) mass loss rates $\dot{M}$ as a function of stellar mass $M_{*}$, disk radius $r_{d}$, and FUV radiation field $G_{0}$. We also provide a simple analytic solution that approximately shows the scaling of the mass loss rate with these parameters. However, the analytic results are presented in terms of the column density $N_{C}$ of the heated surface gas, which is assumed 
to be isothermal with sound speed $a_{s}$. Comparison to PDR codes is required to determine $N_{C}$ and $a_{s}$ for a given radiation field $G_{0}$.

[6] The mass loss rate is significant for disk radii much smaller than the critical radius, in particular for $r_{d} / r_{g} \gtrsim 0.15$. Previous work assumed negligible mass loss for $r_{d}<r_{g}$, so this finding increases the range of viable parameter space for mass loss. However, the mass loss rate drops exponentially for $r_{d} \lesssim 0.15 r_{g}$, scaling roughly as $\dot{M} \propto \exp \left[-r_{g} / 2 r_{d}\right]$.

[7] If a disk has enough viscosity, then viscous spreading of the outer disk edge can affect photoevaporation. As a disk becomes smaller in radius, its photoevaporation time increases whereas its viscous spreading time decreases. As a result, disks will shrink down to the size at which the two time scales are in balance (see Figure 8). This process tends to enhance the effectiveness of photoevaporation by feeding new material into the outer disk where it can be efficiently removed by the outflow.

[8] Photoevaporation is most effective for disks surrounding stars of low mass ( $§ 7)$. For example, a disk around an M dwarf with $M_{*}=0.25 M_{\odot}$ can be evaporated down to $10 \mathrm{AU}$ in only $12 \mathrm{Myr}$ when exposed to a modest FUV radiation field with $G_{0}=3000$. Such radiation intensities occur readily in moderately sized stellar groups, those with $N_{\star} \sim 300$, which represent a common star forming environment (e.g., Lada \& Lada 2003, Porras et al. 2003).

A intriguing result emerges from this consideration of disk evaporation and the corresponding loss of planet forming potential for stars with varying mass. High mass stars are efficient at evaporating their own circumstellar disks and are thus not expected to harbor planets. At the other end of the mass spectrum, red dwarfs easily lose their disks due to photoevaporation in the presence of modest external FUV radiation fields (e.g., $G_{0}=3000$ ), which are expected in common star forming units. As a result, solar type stars (loosely speaking, stars with masses within a factor of two of $\left.1.0 M_{\odot}\right)$ are the preferred locations for giant planet formation.

\section{Acknowledgments}

We would like to thank M. Kaufman and A. Parravano for useful discussions. We also thank an anonymous referee for comments that clarified the paper. This work was supported by a grant from the NASA Origins of the Solar System Program, the NASA Astrophysics Theory Program, and by the Michigan Center for Theoretical Physics. 


\section{APPENDIX: ANALYTIC APPROXIMATION FOR THE PHOTOEVAPORATION OF SMALL DISKS $\left(r_{d} \ll r_{g}\right)$}

In this Appendix, we derive simple analytic results for the scaling of the photoevaporative mass loss rate $\dot{M}$ as a function of stellar mass $M_{*}$, disk radius $r_{d}$, and (implicitly) the strength of the FUV radiation field $G_{0}$. Specifically, we make the following simplifying assumptions:

[i] The gas is essentially static in the inner region where $r<r_{s}$, with thermal pressure balancing gravity. This assumption is equivalent to neglecting the $v d v / d r$ term in equation (18) and solving the remaining equation for the density structure $n(r)$.

[ii] The outflow velocity $v$ is constant in the outer region where $r>r_{s}$, with $v=a_{s}$, the sound speed at the sonic point. This assumption implies that the density profile in the outer region has the form $n(r)=n_{s}\left(r_{s} / r\right)^{2}$, where $n_{s}$ is the number density at $r_{s}$.

[iii] The FUV field $G_{0}$ heats a column $N_{C}$ of surface gas to a constant temperature $T_{s}$ (i.e., the surface layer is isothermal). We thus obtain results that depend on $T_{s}\left(a_{s}\right)$ and $N_{C}$, but these parameters are actually surrogates for the radiation field $G_{0}$. We can relate $a_{s}$ and $N_{C}$ to $G_{0}$ using the results from the PDR code as shown in Figure 2.

Figure 1 shows a schematic representation of the photoevaporation process for subcritical disks. Here, we work in the limit $r_{d} \ll r_{g}$ and assume that the critical radius $r_{g}$ and the sonic radius $r_{s}$ are comparable $\left(r_{s} \sim r_{g}\right)$. With this set of approximations, the density profile for the subsonic region takes the form

$$
n(r)=n_{d} \exp \left[-\frac{r_{g}}{2 r_{d}}\left(1-r_{d} / r\right)^{2}\right]
$$

where $r_{g}$ is the critical radius (given by eq. [1]) with $T=T_{s}$. Assuming that $r_{s}, r_{g} \gg r_{d}$, we thus obtain

$$
n_{s} \approx n_{d} \exp \left[-r_{g} / 2 r_{d}\right]
$$

The mass loss rate from the disk edge $\left(\right.$ at $r_{d}$ ) is given by the continuity equation and takes the form

$$
\dot{M}=\langle\mu\rangle n_{s} a_{s} \mathcal{A}_{s}
$$

where $\langle\mu\rangle$ is the mass per particle and where $\mathcal{A}_{s}$ is the area subtended by the flow at $r_{s}$. This area can be written

$$
\mathcal{A}_{s}=2 \pi r_{d} H_{d}\left(r_{s} / r_{d}\right)^{2} \equiv 2 \pi \alpha r_{g}\left(r_{d} r_{g}\right)^{1 / 2}
$$

In the second equality, we have evaluated the disk scale height $H_{d}=r_{g}\left(r_{d} / r_{g}\right)^{3 / 2}$ and have defined a dimensionless constant $\alpha \equiv\left(r_{s} / r_{g}\right)^{2}$, which is of order unity. Finally, we apply the condition that the external FUV flux $G_{0}$ heats a column density $N_{C}$ given by the integral

$$
N_{C}=\int_{r_{d}}^{\infty} n(r) d r .
$$


In the limit that $r_{d} \ll r_{g}$, most of the support of this integral occurs for small $r$ where equation (A1) applies. This condition (A5) relates the column density $N_{C}$ to $n_{d}$, and, to leading order, this relation takes the form

$$
n_{d} \approx\left(\frac{2}{\pi}\right)^{1 / 2}\left(\frac{r_{g}}{r_{d}}\right)^{1 / 2} \frac{N_{C}}{r_{d}}
$$

Collecting all of the results given above, we obtain the following expression for the mass loss rate

$$
\dot{M}=C_{0} N_{C}\langle\mu\rangle a_{s} r_{g}\left(\frac{r_{g}}{r_{d}}\right) \mathrm{e}^{-r_{g} / 2 r_{d}},
$$

where $C_{0}$ is a dimensionless constant of order unity.

Although the derivation of equation (A7) applies only in the limit $r_{d} \ll r_{s} \sim r_{g}$, the resulting function can be evaluated when $r_{d} \approx r_{s} \approx r_{g}$ and implies nearly the same result as the supercritical mass loss rate of $\S 3$ (see eq. [9]). Therefore, we can use equation (A7) as an analytic approximation to the mass loss rate (for a given radiation field $G_{0}$ ) as a function of $r_{d} / r_{g}$ (for $r_{d} / r_{g} \leq 1$ ). This approximation should match onto the subcritical mass loss rates calculated in $\S 4$ (where $r_{d} / r_{g} \approx 0.125$ ) and should also match onto the supercritical mass loss rates of $\S 3$ (for $r_{d} \rightarrow r_{g}$ ). Notice that we are implicitly assuming that $a_{s}$ and $N_{C}$ do not change with $r_{d} / r_{g}$ for a given radiation field.

With these approximations, we can estimate the mass loss rates for systems that are intermediate between the subcritical regime of $\S 4$ and the supercritical regime of $\S 3$. We can also use the resulting form of $\dot{M}$ to understand how the mass loss rate depends on the various parameters in the problem. As $r_{d}$ becomes comparable to $r_{g}$, the mass loss rate approach its supercritical value. As $r_{d} / r_{g}$ decreases, the mass loss rate decreases, but only slowly at first. The outflow rate $\dot{M}$ is half its supercritical value when $r_{d} / r_{g} \approx 0.17$ (significantly below unity). For even smaller values of $r_{d} / r_{g}$, however, the decaying exponential behavior wins and the mass loss rates drop dramatically.

Finally, we can also make an analytic estimate for the mass loss rate from the disk surface, i.e., for vertical flow off the top and bottom of the disk. This estimate can be compared to that for mass loss from the disk edges (see eq. [A7]). For vertical flow, we treat each increment of disk surface area $2 \pi r d r$ with the same formulation used above for the disk edges, with one exception: We must replace the radius $r_{d}$ with $r \leq r_{d}$ and then integrate over $r$. This procedure takes into account the fact that material at $r<r_{d}$ lives deeper in the gravitational potential well and is harder to extract from the system. The resulting mass loss rate from the disk surface is

$$
\dot{M}_{s u r}=C_{1} N_{C}\langle\mu\rangle a_{s} r_{g}\left(\frac{r_{g}}{r_{d}}\right)^{1 / 2} \mathrm{e}^{-r_{g} / 2 r_{d}}
$$

where all of the dimensionless quantities are collected into the constant $C_{1}$ (which is comparable to, but not quite the same as, the constant $C_{0}$ appearing in eq. [A7]). Comparing the mass loss rates from the disk edge and the disk surface, we find that $\dot{M}_{\text {sur }} / \dot{M} \approx\left(r_{d} / r_{g}\right)^{1 / 2}$. In the limit $r_{d} / r_{g} \ll 1$, the mass loss rate from the disk edge dominates the mass loss rate from the disk surfaces. 


\section{REFERENCES}

Adams, F. C. 2000, ApJ, 542, 964

Adams, F. C. \& Laughlin, G. 2001, Icarus, 150, 151

Adams, F. C., \& Myers, P. C. 2001, ApJ, 553, 744

Allen, R. L., Bernstein, G. M.,\& Malhotra, R. 2002, AJ, 124, 2949

Armitage, P. J. 2000, A\&A, 362, 968

Backman, D., Gillett, F., \& Witteborn, F. 1992, ApJ, 385, 670

Bally, J., O’Dell, C. R., \& McCaughrean, M. J. 2000, AJ, 119, 2919

Bally, J., Sutherland, R.S., Devine, D., Johnstone, D. 1998, AJ, 116, 293

Begelman, M. C., McKee, C. F., \& Shields, G. A. 1983, ApJ, 271, 70

Bertoldi, G., \& Draine, B. T. 1996, 458, 222

Bodenheimer, P., \& Pollack, J. B. 1986, Icarus, 67, 391

Boss, A. P., \& Foster, P. N. 1998, ApJ, 494, L103

Boss, A. P. 2000, ApJ, 536, L101

Cameron, A.G.W., Hoeflich, P., Myers, P. C., \& Cayton, D. D. 1995, ApJ, 447, L53

Carpenter, J. 2000, AJ, 120, 3139

Carpenter, J. M. 2002, AJ, 124, 1593

Chiang, E. I., Fischer, D., \& Thommes, E. 2002, ApJ, 564, 105

Churchwell, E., Wood, D., Felli, M., \& Massi, M. 1987, ApJ, 321, 516

Clarke, C. J., Gendrin, A., \& Sotomayor, M. 2001, MNRAS, 328, 485

Cochran, W. D., Hatzes, A. P., \& Paulson, D. B. 2002, AJ, 124, 565

d'Alessio, P., et al. 1999, ApJ, 527, 893

Gilliland, R. L., et al. 2000, ApJ, 545, 47

Gonzalez, G., Law, C., Tyagi, S., \& Reddy, B. E. 2001, AJ, 121, 432

Haisch, K. E., et al. 2001, AJ, 121, 1512

Hatzes, A. P., \& Cochran, W. D. 2000, Proc. SPIE, 4005, 252 
Hillenbrand, L. A., \& Hartmann, L. W. 1999, ApJ, 492, 540

Hollenbach, D., \& Adams, F. C. 2003, in The Gillett Symposium: Debris Disks and the Formation of Planets, eds. D. Backman \& L. Caroff (San Francisco: ASP Publishing), in press

Hollenbach, D., Johnstone, D., Lizano, S., \& Shu, F. H. 1994, ApJ, 428, 654

Hollenbach, D., \& Tielens, A.G.G.M. 1999, RvMP, 71, 173

Hollenbach, D., Yorke, H., \& Johnstone, D. 2000, Protostars and Planets IV, ed. V. Mannings (Tucson: Univ. Arizona Press), 401

Johnstone, D., Hollenbach, D., Bally, J. 1998, ApJ, 499, 758

Kaufman, M., Wolfire, M., Hollenbach, D., \& Luhman, M. 1999, ApJ, 527, 795

Kominami, J., \& Ida, S. 2002, Icarus, 159, 322

Kroupa, P. 1995, MNRAS, 277, 1522

Lada, C. J., \& Lada, E. A. 2003, ARA\&A, 41, 57

Liffman, K. 2003, PASA, 20, 337L

Lin, D.N.C., \& Papaloizou, J.C.B. 1986, ApJ, 309, 846

Lissauer, J. J. 1993, ARA\&A, 31, 129

Matsuyama, I., Johnstone, D., \& Hartmann, L. 2003a, ApJ, 582, 893

Matsuyama, I., Johnstone, D., \& Murray, N. 2003b, ApJ, 585, 143

McCaughrean, M. J. \& O’Dell, C. R., 1996, AJ, 111, 1977

Mochejska, B. J., Stanek, K. Z., Sasselov, D. D., \& Szentgyorgyi, A. H. 2002, AJ, 123, 3460

O’Dell, C.R. 1998, AJ, 115, 263

Parravano, A., Hollenbach, D. J., \& McKee, C. F. 2003, ApJ, 584, 797

Paulson, D., Saar, S., Cochran, W., \& Henry, G. 2004, AJ, in press

Perryman, M.A.C., et al. 1998, A\&A, 331, 81

Porras, A., Christopher, M., Allen, L., DiFrancesco, J., Megeath, S. T., \& Myers, P. C. 2003, AJ, 126,1916

Richling, S., Yorke, H. W. 1997, A\&A, 327, 317

Richling, S. \& Yorke, H. 1998, A\&A, 340, 508 
Richling, S. \& Yorke, H. 2000, ApJ, 539, 258

Salpeter, E. E. 1955, ApJ, 121, 161

Scally, A., \& Clarke, C. 2001, MNRAS, 325, 449

Shu, F. H., Johnstone, D., \& Hollenbach, D. 1993, Icarus, 106, 92

Störzer, H., \& Hollenbach, D. 1998, ApJ, 495, 853

Störzer, H., \& Hollenbach, D. 1999, ApJ, 515, 669 (SH99)

Suchkov, A. A., \& Schultz, A. B. 2001, ApJ, 549, L237

Tanaka, H., \& Ida, S. 1997, Icarus, 125, 302

Tielens, A.G.G.M., \& Hollenbach, D. 1985, ApJ, 291, 722

Testi, L., Palla, F., Prusti, T., Natta, A., \& Maltagliati, S. 1997, A\&A, 370, 159

Testi, L., Palla, F., \& Natta, A. 1998, A\&A Suppl., 133, 81

Testi, L., Palla, F., \& Natta, A. 1999, A\&A, 342, 515

Thommes, E. W., Duncan, M. J., \& Levison, H. F. 1999, Nature, 402, 635

Throop, H. B., Bally, J., Esposito, L. W., McCaughrean, M. J. 2001, Science, 292, 1686

Trujillo, C. A. \& Brown, M. E. 2001, ApJ, 554, L95

Yorke, H. \& Richling, S. 2002, RevMxAC, 12, 92

Ward, W. R. 1997, Icarus, 126, 261

Weidenschilling, S. J. 1997, Icarus, 127, 290

Woods, D. T., et al. 1996, ApJ, 461, 767

Zuckerman, B., Forveille, T., \& Kastner, J. H. 1995, Nature, 373, 494 


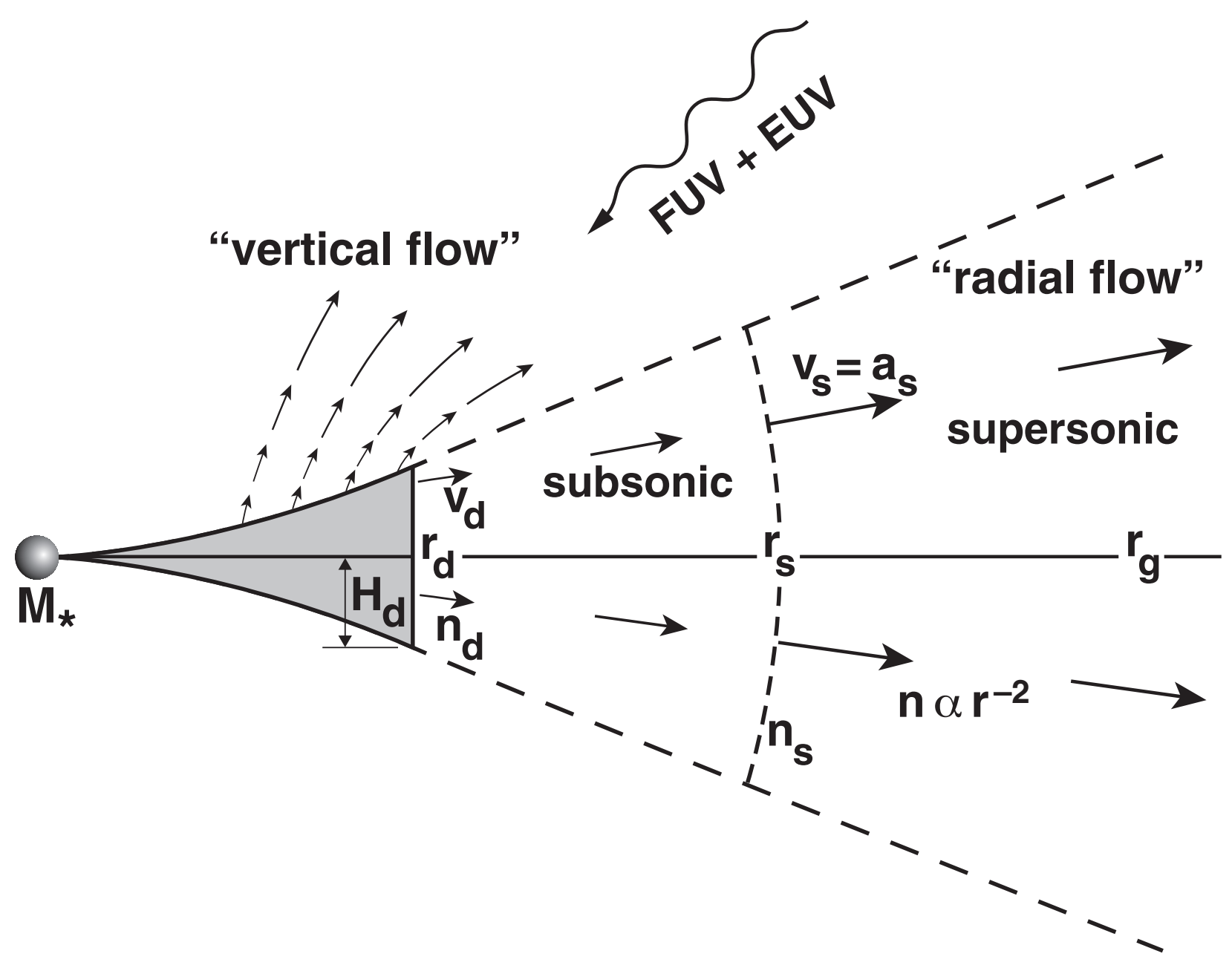

Fig. 1.- Schematic of a disk with radius $r_{d}$ around a star with mass $M_{*}$, illuminated by the FUV (and perhaps EUV) radiation from nearby stars of greater mass. The disk is inclined so that the top and edge are exposed. The disk scale height is $H_{d}$ at the outer radius $r_{d}$. In the subcritical regime, where $r_{d}<r_{g}$, the bulk of the photoevaporation flow (the radial flow) originates from the disk edge, which marks the inner boundary. The flow begins subsonically at $r_{d}$, with speed $v_{d}$ and density $n_{d}$. The flow accelerates to the sound speed at $r_{s}$ (the sonic point), which lies inside the critical escape radius $r_{g}$. Beyond the sonic point, the flow attains a terminal speed of order the sound speed and the density falls roughly as $n \propto r^{-2}$. Although some material is lost off the top and bottom faces of the disk (the vertical flow), its contribution to the mass loss rate is secondary to that from the edges. Nonetheless, the polar regions are not evacuated, the star is fully enveloped by circumstellar material, and the incoming FUV radiation will be attenuated in all directions. 


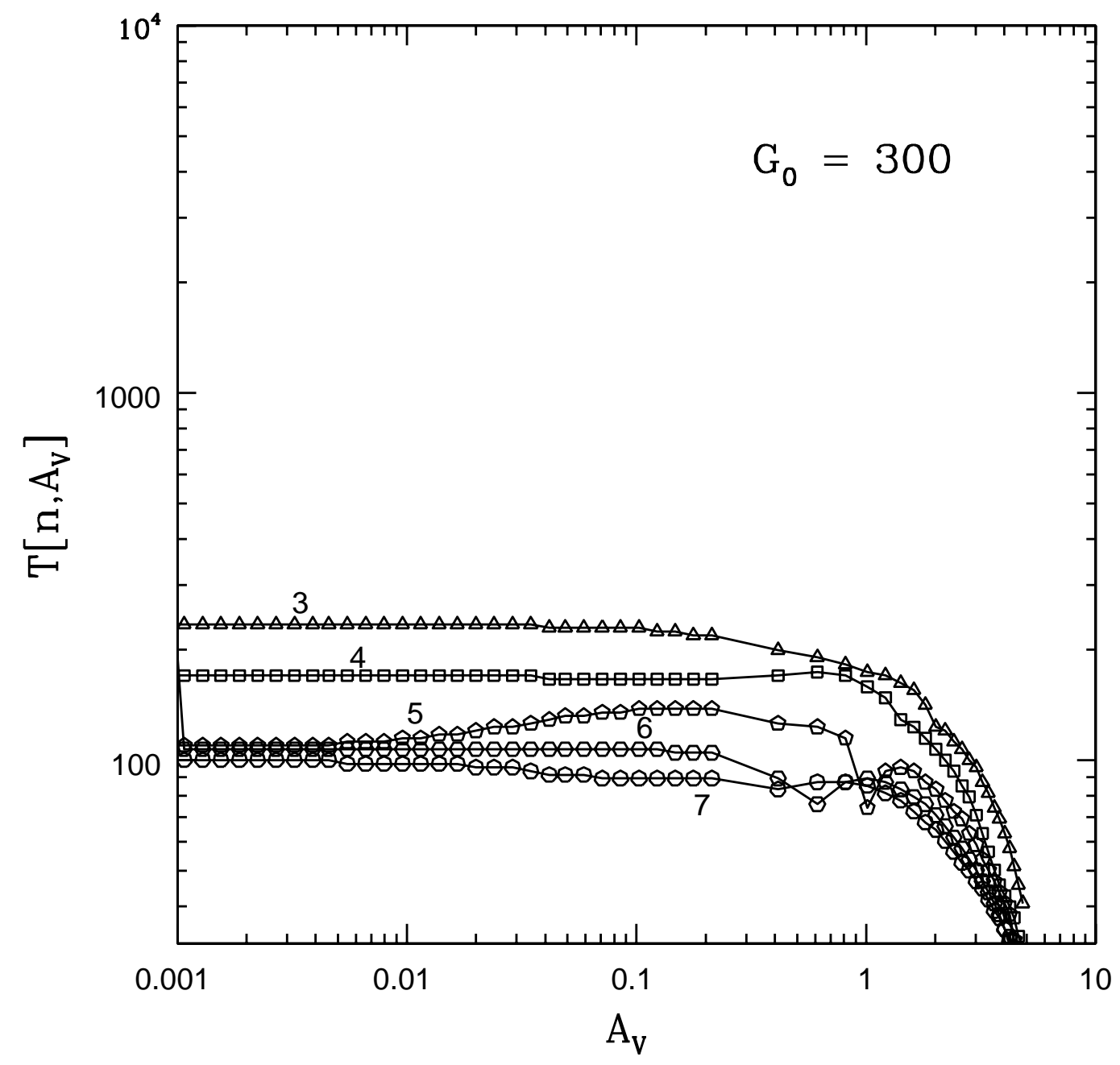

Fig. 2a.- Temperature profiles calculated from the PDR code for an external FUV radiation field. Each curve shows the temperature as a function of visual extinction $A_{V}$ for a given number density $n=10^{p} \mathrm{~cm}^{-3}$, shown here for $p=3-8$. The values of $p$ are labeled for each curve; in addition, each curve is marked by polygons, where the number of sides corresponds to the value of $p$. (a) Results for $G_{0}=300$. (b) Results for $G_{0}=3000$. (c) Results for $G_{0}=30,000$. 


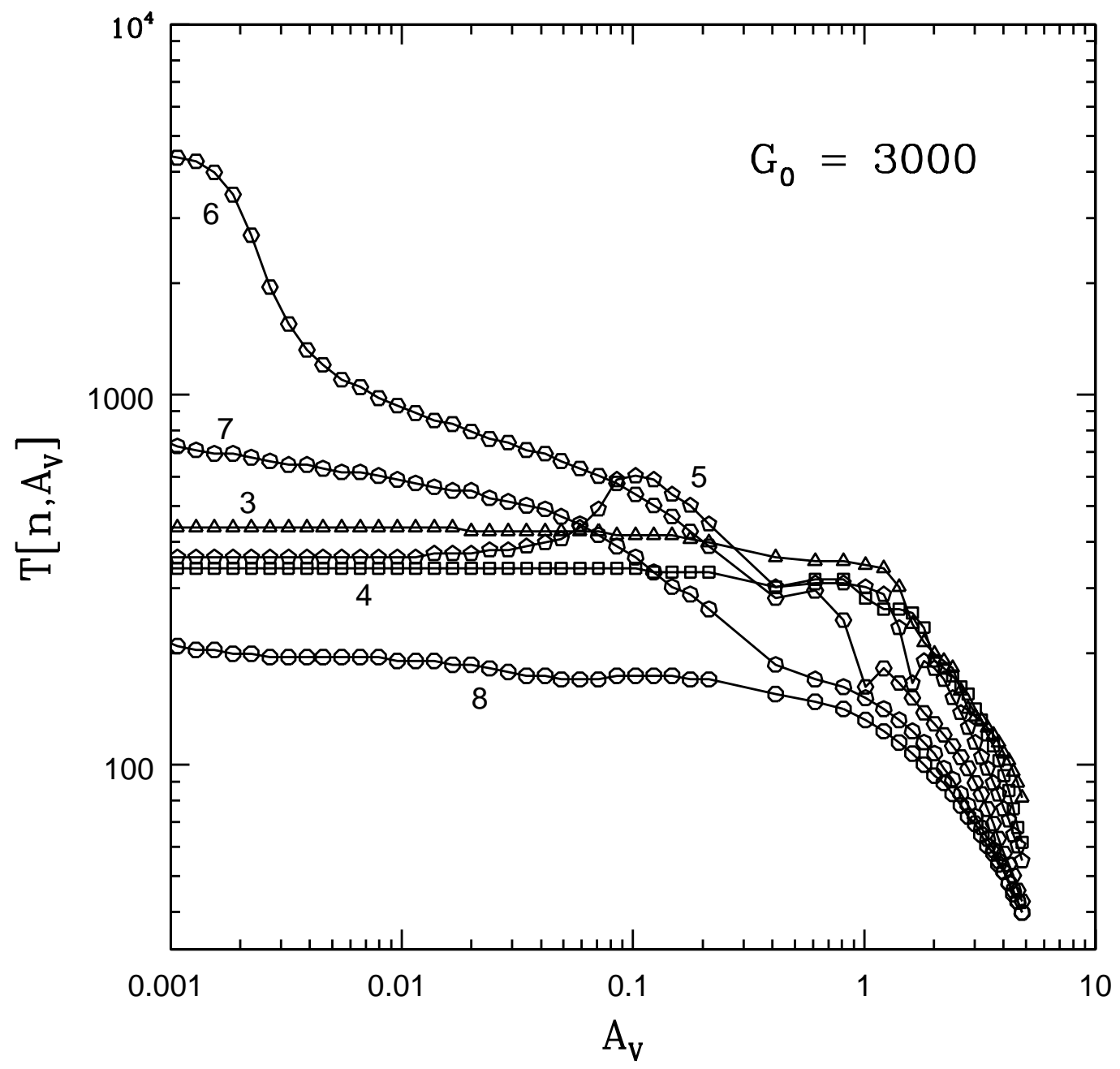




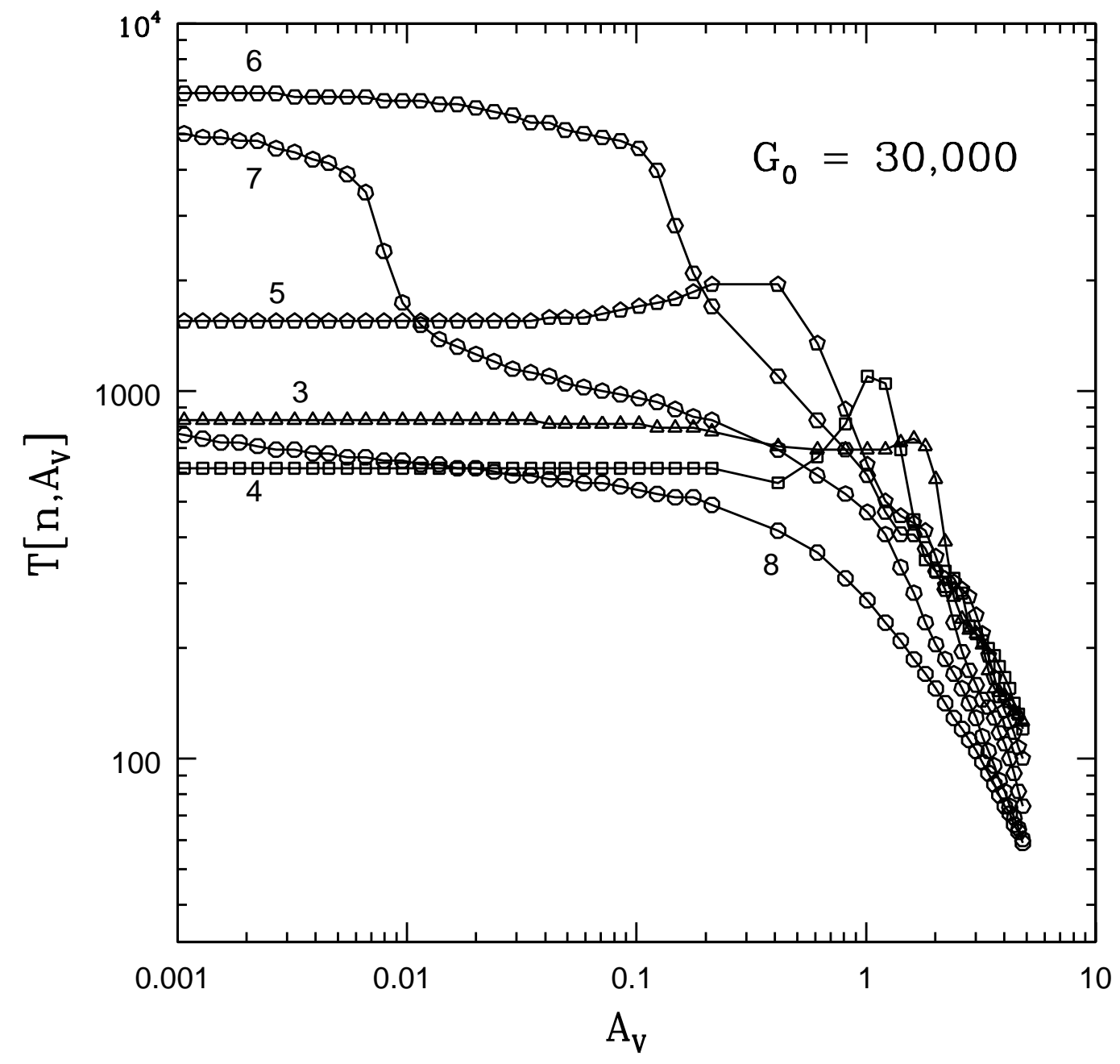




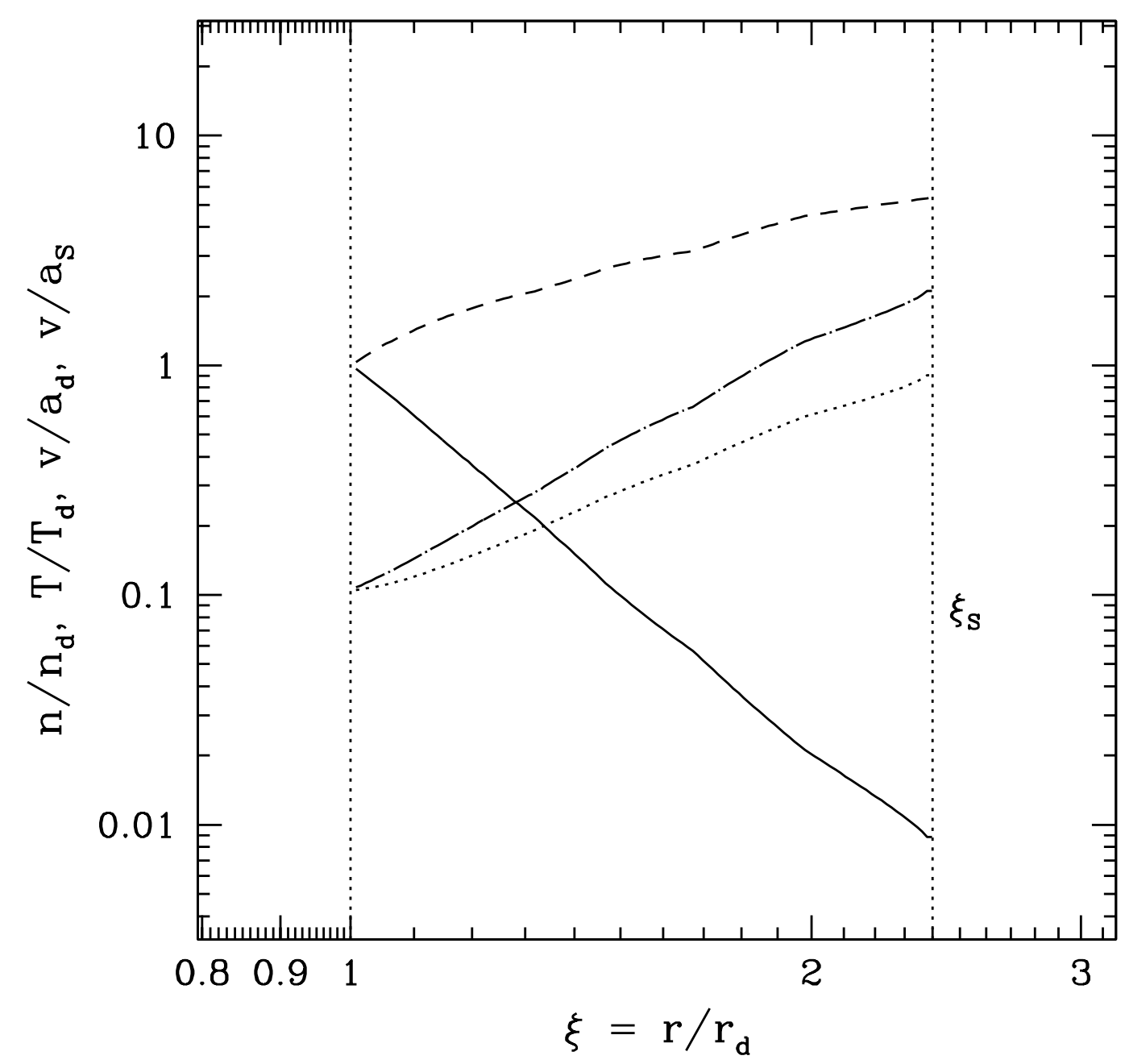

Fig. 3.- Radial profiles of the fluid fields for a disk with $r_{d}=30$ AU surrounding a solar mass star $\left(M_{*}=1.0 M_{\odot}\right)$, where the radiation field has intensity $G_{0}=3000$. The solid curve shows the run of density; the dashed curve shows the temperature; the dot-dashed curve shows the outflow speed. All of these quantities are normalized to their values at the outer disk edge. Also shown, depicted as the dotted curve, is the Mach number $\mathcal{M}=v / a_{S}$, which reaches unity at the sonic point (by definition). Finally, the vertical lines at $\xi=1, \xi_{s}$ mark the locations of the disk edge and the sonic point, respectively. 


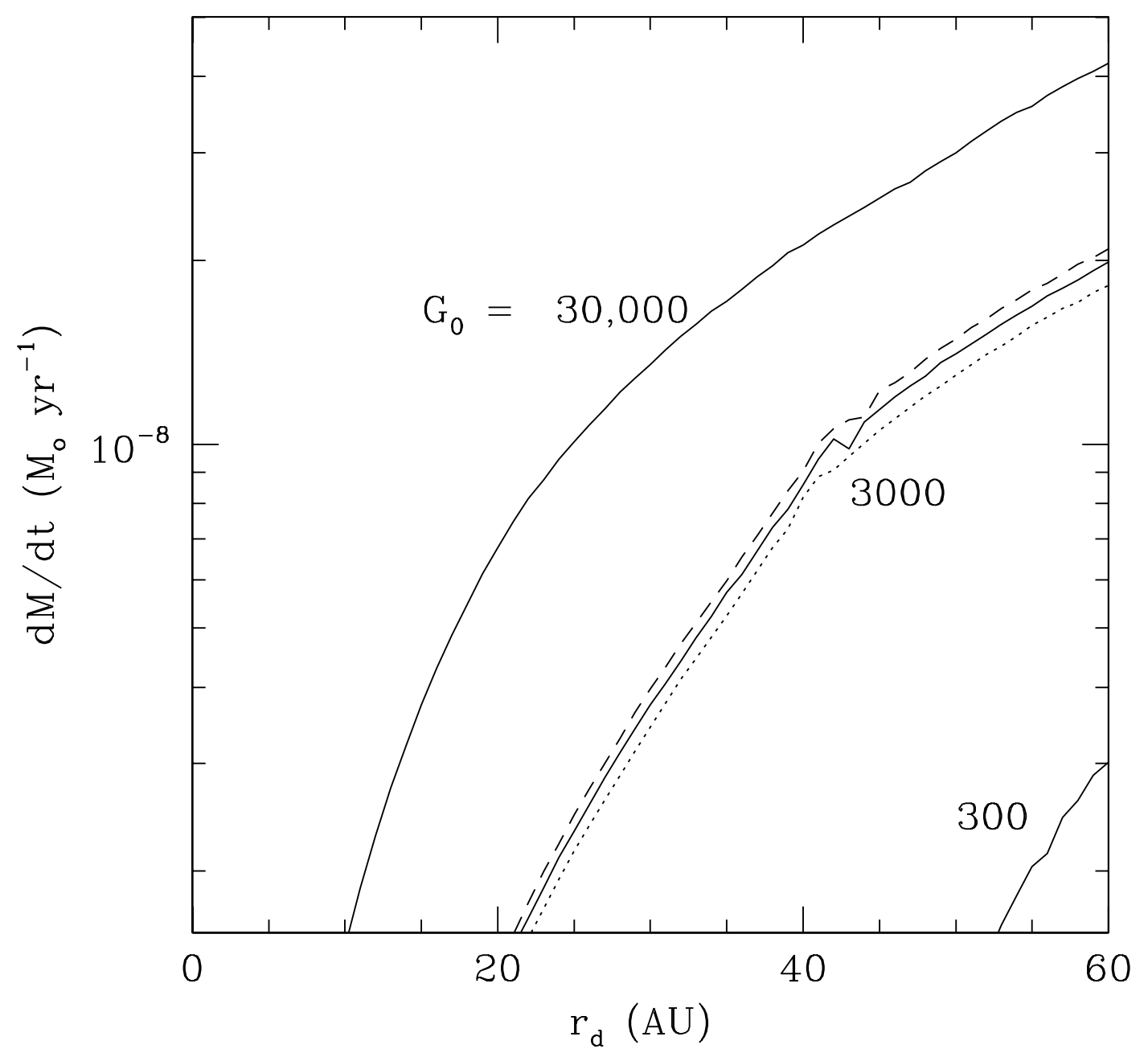

Fig. 4. - The mass loss rates due to photoevaporation for a circumstellar disk embedded in FUV radiation fields of varying intensities (as labeled). The mass loss rates are shown as a function of disk radius $r_{d}$ for a fixed stellar mass $M_{*}=1.0 M_{\odot}$. For the radiation field with intensity $G_{0}$ $=3000$, we show the effects of varying the inner boundary condition. The solid curve uses our standard choice of inner boundary condition where $T_{d}=75 \mathrm{~K}$ at $r_{d}=30 \mathrm{AU}$; the dashed curve shows the alternate choice of $T_{d}(30 \mathrm{AU})=90 \mathrm{~K}$; the dotted curve uses $T_{d}(30 \mathrm{AU})=60 \mathrm{~K}$. In all cases, the disk "surface" temperature (in the absence of external flux) is assumed to follow a power-law of the form $T_{d}=T_{d}(30 \mathrm{AU})(30 \mathrm{AU} / r)^{1 / 2}$. 


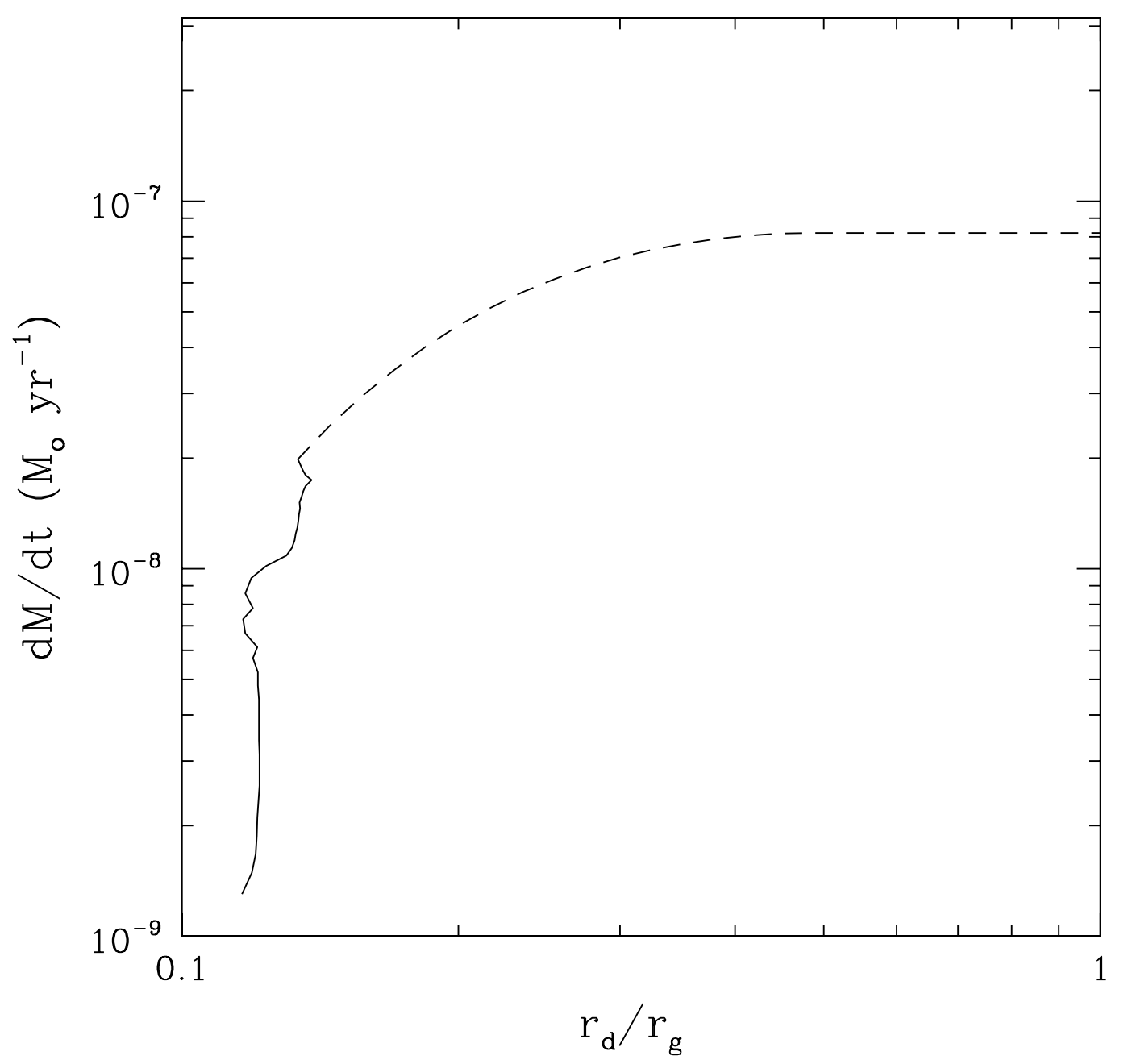

Fig. 5. - The mass loss rate (in $M_{\odot} \mathrm{yr}^{-1}$ ) is shown as a function of $r_{d} / r_{g}$ for a disk surrounding a $M_{*}=1.0 M_{\odot}$ star with an external FUV radiation $G_{0}=3000$. The solid portion of the curve shows results from our numerical treatment, which spans a wide range in $\dot{M}$, but a relatively narrow range in $r_{d} / r_{g}$. The dashed portion of the curve shows the analytic estimate (see the Appendix), which smoothly joins that numerical result (for subcritical disks) onto the result for supercritical disks. The numerical results do not follow a smooth curve because the temperature $T_{s}$ at the sonic point (and hence $r_{g}$ ) depends on $r_{d}$ through the complicated PDR dependence of $T_{s}$ on $n$ and $N_{H}$ (see Fig. 2). Here we assume a constant $r_{g}$ for the analytic (dashed) portion of the curve. 


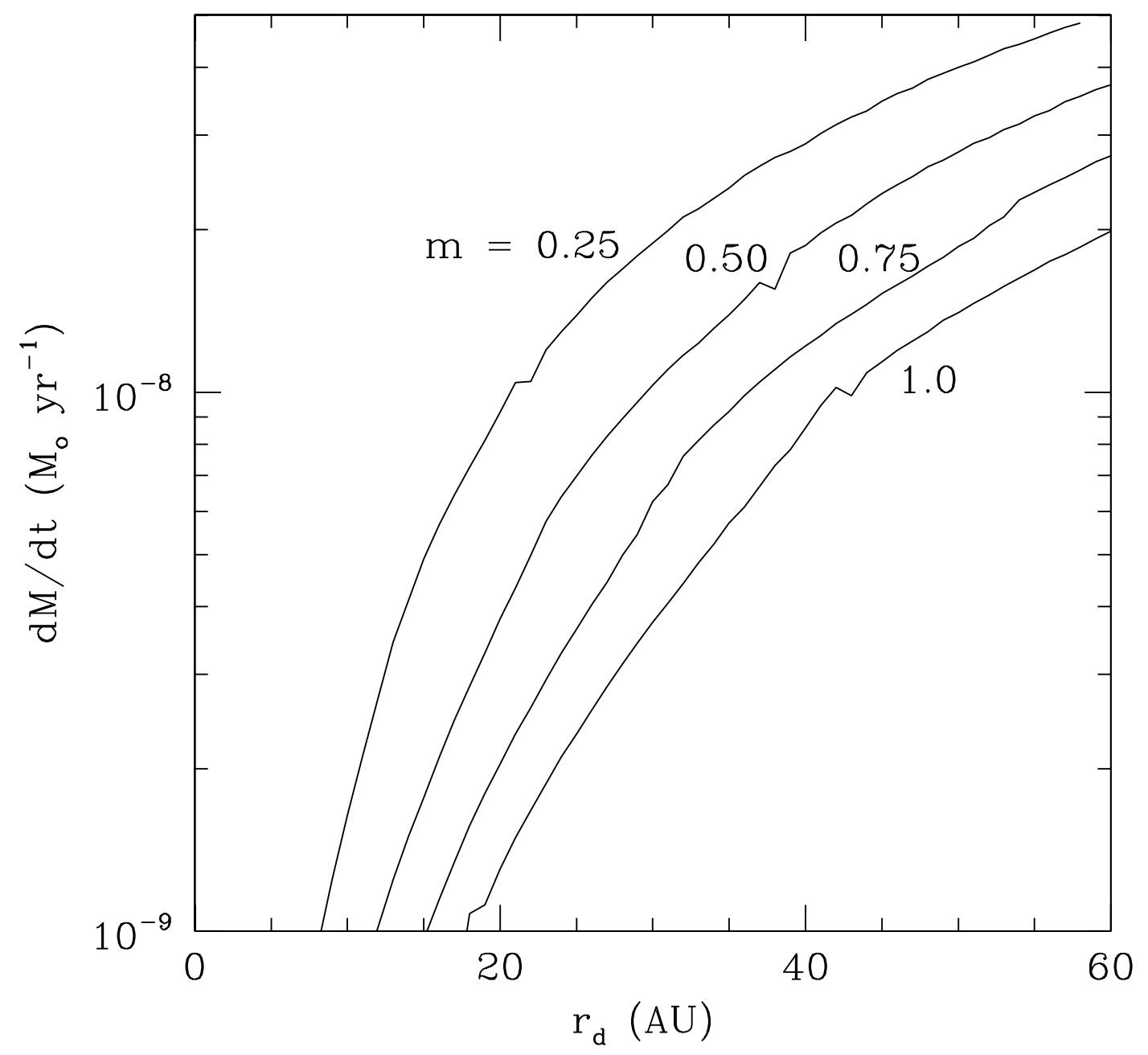

Fig. 6. - The mass loss rates due to photoevaporation for circumstellar disks embedded in an FUV radiation field with $G_{0}=3000$. The mass loss rates are shown as a function of disk radius $r_{d}$ for varying masses of the central stars, where $m=M_{*} / M_{\odot}=0.25,0.50,0.75$ and 1.0 (as labeled). 


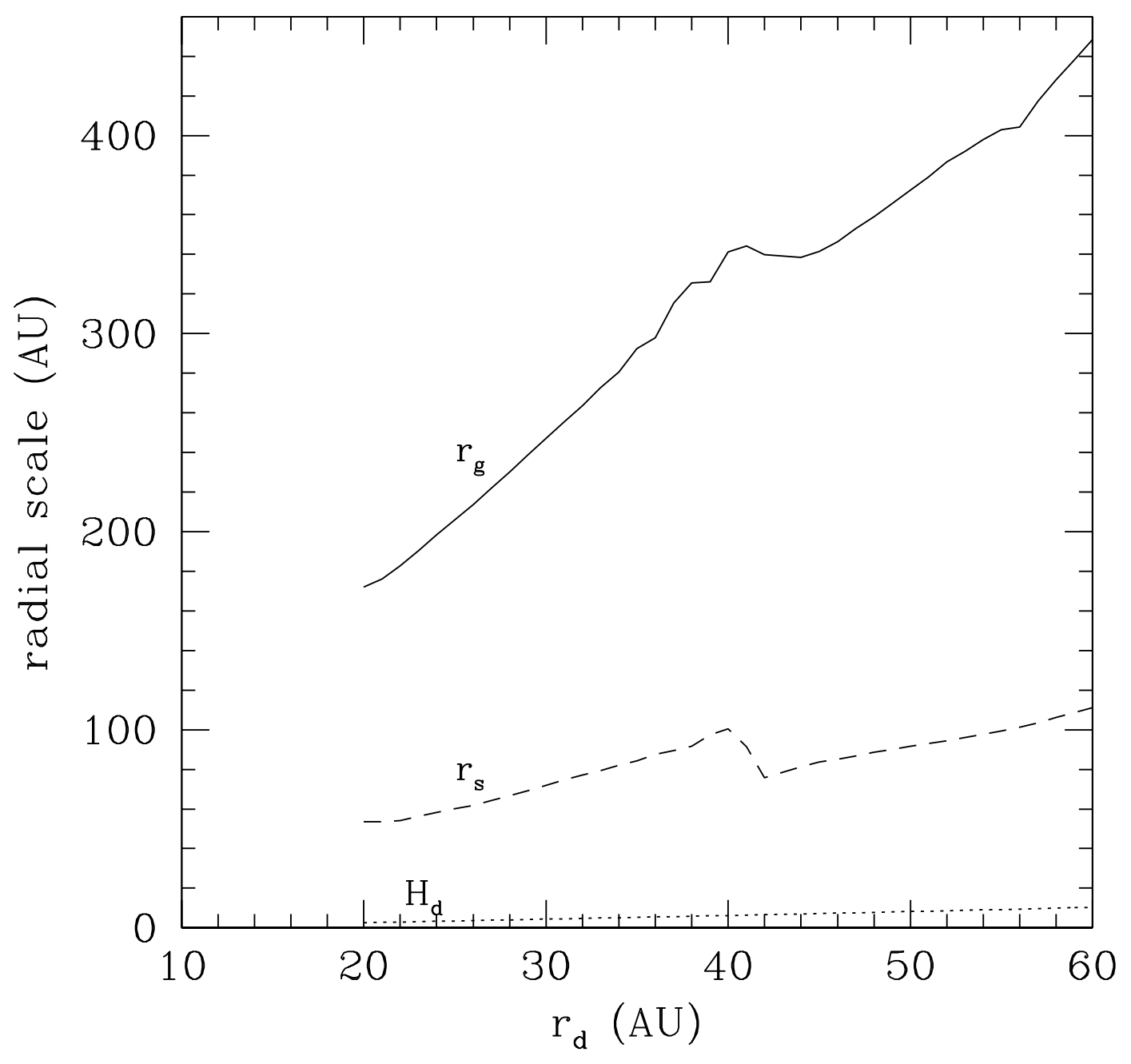

Fig. 7.- This figure shows the relevant length scales as a function of disk radius $r_{d}$ for a circumstellar disk in orbit about a star with mass $M_{*}=1.0 M_{\odot}$ and exposed to an FUV radiation field of intensity $G_{0}=3000$. The dotted curve shows the disk scale height $H_{d}$. The dashed curve shows the sonic radius $r_{s}$ as a function of disk radius. The solid curve shows the critical radius $r_{g}$. The structure in the latter two curves is a reflection of the structure in the relationship between temperature and visual extinction (see Fig. 2). 


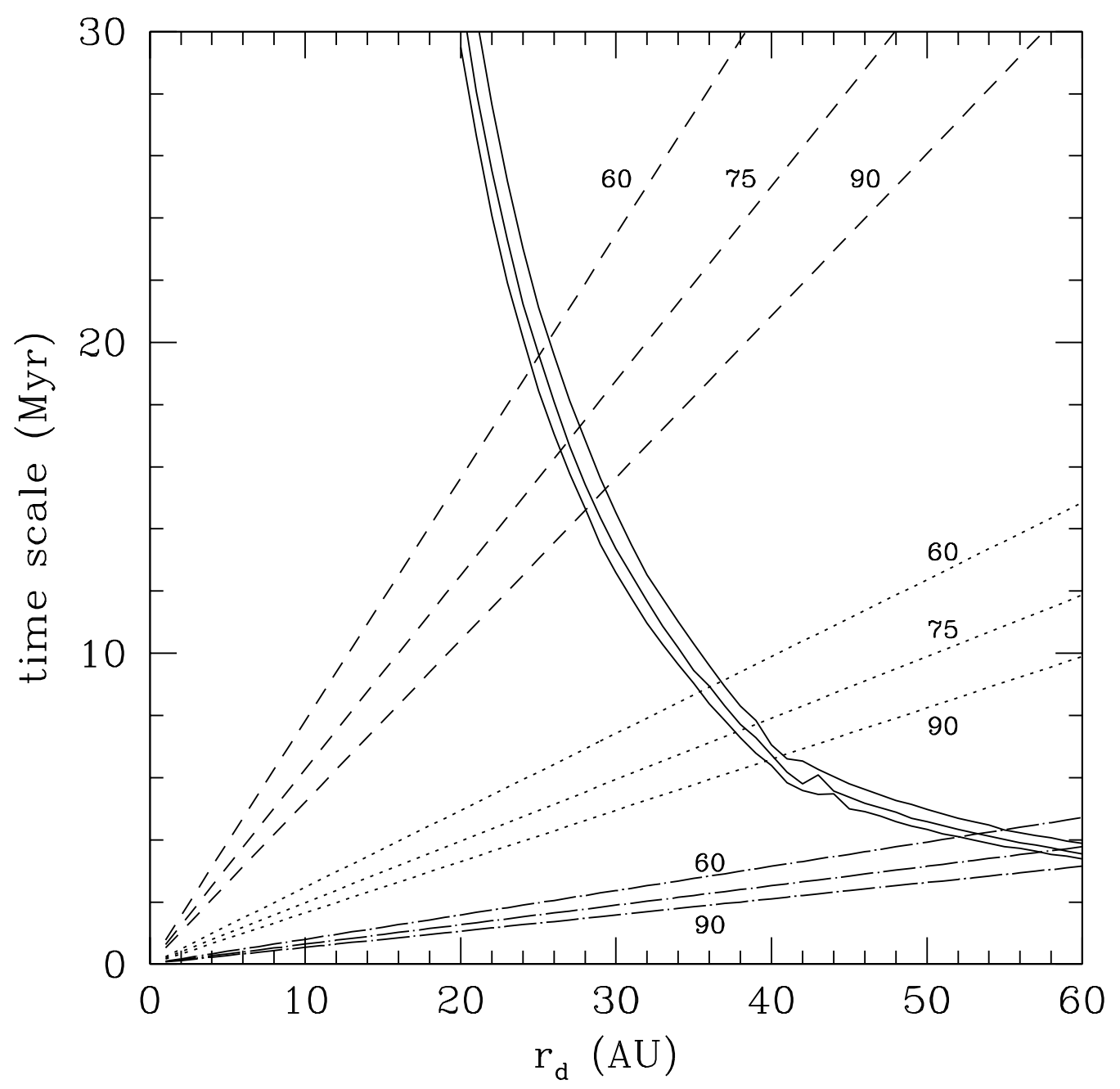

Fig. 8.- Comparison of evaporation time scales and disk accretion time scales for a disk surrounding a star with mass $M_{*}=1.0 M_{\odot}$. The FUV radiation field has $G_{0}=3000$. The solid curve shows the evaporation time scale, as calculated in this paper, which is a decreasing function of disk radius. The disk accretion time is an increasing function of disk radius. Results are shown for viscosity parameter $\alpha=10^{-4}$ (dashed lines), $\alpha=10^{-3.5}$ (dotted lines), and $\alpha=10^{-3}$ (dot-dashed lines). Each case has three separate curves which correspond to different choices for the disk temperature scale, i.e., the temperature $T_{d}\left(r_{d}=30 \mathrm{AU}\right)$; the labels refer to $T_{d}(30 \mathrm{AU})$ in Kelvin. For a given value of the viscosity parameter $\alpha$, the disk will shrink down to the radius where the evaporation time equals the accretion time. 


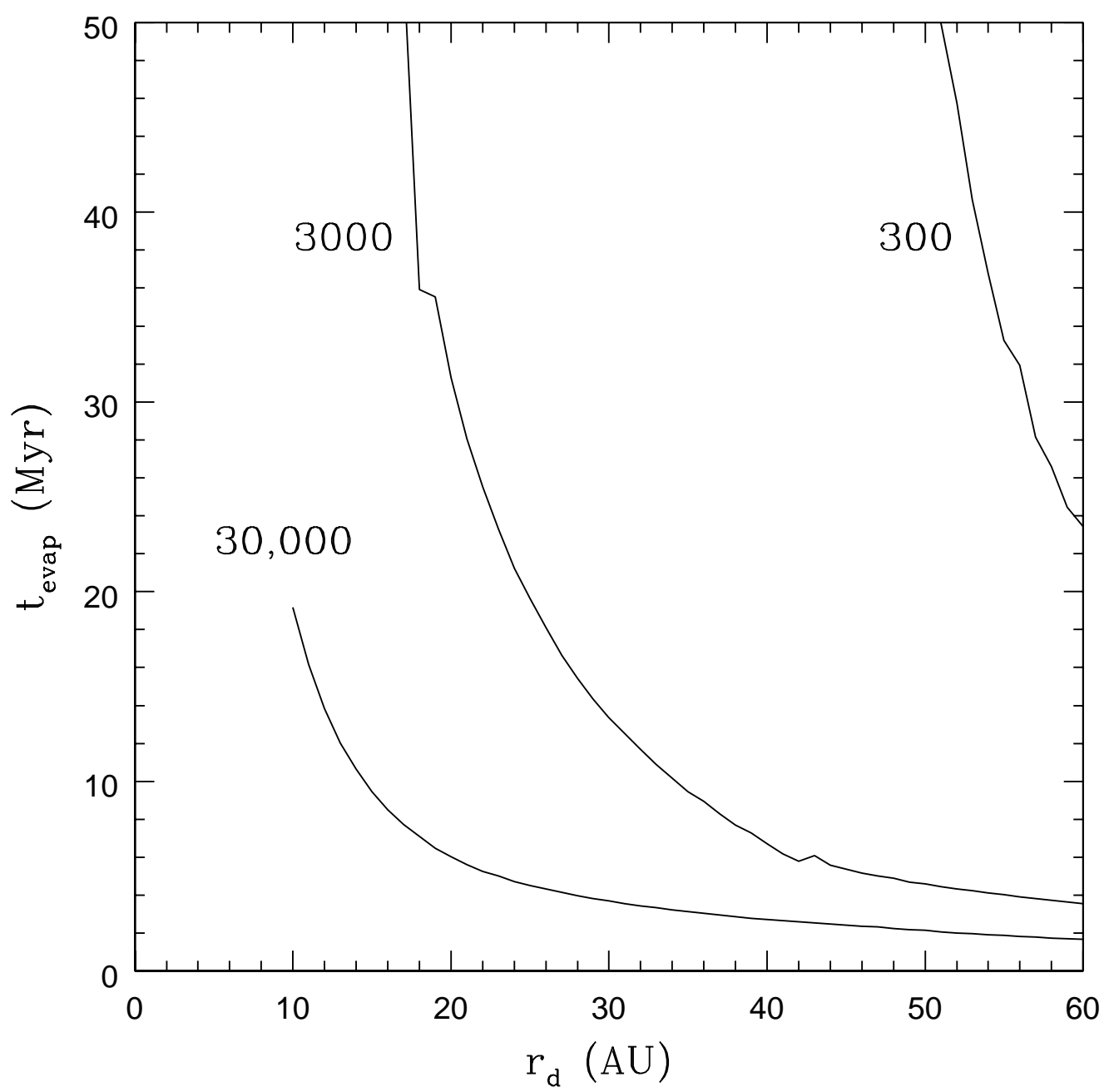

Fig. 9.- Photoevaporation time scales for circumstellar disks exposed to varying external radiation fields, $G_{0}=300,3000$, and 30,000 (as labeled). These models assume that the disk orbit around central stars with mass $M_{*}=1.0 M_{\odot}$. The evaporation rates are calculated according to the formulation of this paper. To specify the evaporation time scale, we assume that disks have masses $M_{d}=0.05 M_{*}\left(r_{d} / 30 \mathrm{AU}\right)^{1 / 2}$. The evaporation time scales, as plotted, are proportional to the assumed disk mass, $t_{\text {evap }} \propto M_{d}$. 


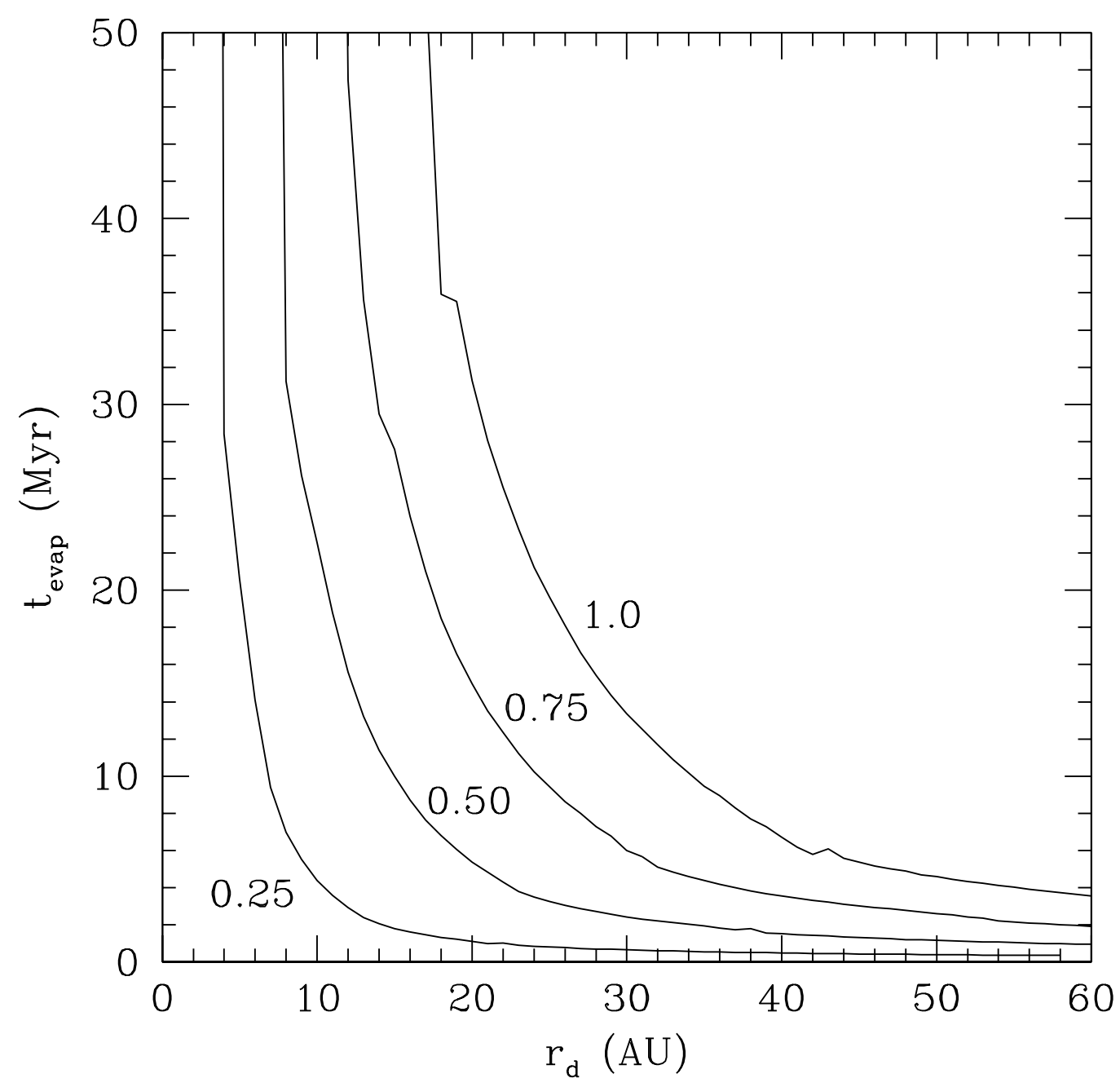

Fig. 10.- Photoevaporation time scales for circumstellar disks surrounding stars with varying masses $m=M_{*} / M_{\odot}=1.0,0.75,0.5$, and 0.25 (as labeled). The external FUV radiation field has intensity $G_{0}=3000$ for all cases shown. The evaporation rates are calculated according to the formulation of this paper. To specify the evaporation time scale, we assume that disks have masses $M_{d}=0.05 M_{*}\left(r_{d} / 30 \mathrm{AU}\right)^{1 / 2}$. The evaporation time scales, as plotted, are proportional to the assumed disk mass, $t_{\text {evap }} \propto M_{d}$. 